\title{
Giant excitonic upconverted emission of two- dimensional semiconductor in doubly resonant plasmonic nanocavity
}

\section{Pengfei Qi}

Peking University

\section{Yuchen Dai}

Peking University

Luo Yang

Peking University

\section{Guangyi Tao}

Peking University

\section{Liheng Zheng}

Peking University

\section{Tianhao Zhang}

Nankai University

\section{Bo Shen}

Peking University

Feng Lin

Peking University

Zheyu Fang ( $\nabla$ zhyfang@pku.edu.cn )

Peking University https://orcid.org/0000-0001-5780-0728

\section{Article}

Keywords: Two-dimensional semiconductor, Upconverted emission, Plasmonic nanocavity

Posted Date: May 3rd, 2021

DOI: https://doi.org/10.21203/rs.3.rs-429365/v1

License: (c) (1) This work is licensed under a Creative Commons Attribution 4.0 International License. Read Full License 


\section{Giant excitonic upconverted emission of two-dimensional} semiconductor in doubly resonant plasmonic nanocavity Shen, ${ }^{1}$ Feng Lin, ${ }^{1}$ Zheyu Fang ${ }^{1, *}$

1 School of Physics, State Key Laboratory for Mesoscopic Physics, Academy for Advanced Interdisciplinary Studies, Collaborative Innovation Center of Quantum Matter, Nano-optoelectronics Frontier Center of Ministry of Education, Peking University, Beijing 100871, China

${ }^{2}$ Photonics Research Center, School of Physics, MOE Key Lab of Weak-Light Nonlinear Photonics, and Tianjin Key Lab of Photonics Materials and Technology for Information Science, Nankai University, Tianjin 300071, China

\# These authors contributed equally: Pengfei Qi, Yuchen Dai and Yang Luo

*Email: zhyfang@pku.edu.cn

Abstract: Phonon-assisted upconverted emission lies at the heart of energy harvesting, bioimaging, optical cryptography and optical refrigeration. It has been demonstrated that the emerging twodimensional (2D) semiconductors can provide a great platform for efficient phonon-assisted upconversion due to the enhanced optical transition strength and phonon-exciton interaction of $2 \mathrm{D}$ excitons. However, the research on the further enhancement of excitonic upconverted emission in 2D semiconductors is almost blank. Here we report the enhanced multiphoton upconverted emission of 2D excitons in doubly resonant plasmonic nanocavity. Owing to the enhanced light collection, enhanced excitation rate and quantum efficiency enhancement arising from Purcell effect, the upconverted emission amplification of $>1000$ folds and the decrease of $2 \sim 3$ orders of magnitude for saturated excitation energy density are achieved. These findings pave the way to the development of excitonic upconversion lasing, nanoscopic thermometry and sensing, and open up the possibility of optical refrigeration in future $2 \mathrm{D}$ electronic or excitonic devices.

Key Words: Two-dimensional semiconductor, Upconverted emission, Plasmonic nanocavity

Photon upconversion is an anti-Stokes process to emit a photon at energy higher than excitation photon energy through a variety of mechanisms, ranging from high harmonic generation, multiphoton absorption, Auger recombination to phonon scattering. ${ }^{1-7}$ The latter relevant to this work, phononassisted anti-Stokes emission has been demonstrated as an appealing possibility for fundamental studies and applications, including bioimaging and phototherapy, ${ }^{8-15}$ volumetric displays, ${ }^{16}$ 
upconversion lasers, ${ }^{17-19}$ optical writing, ${ }^{20}$ optical tweezers, ${ }^{21}$ optical cryocooling, ${ }^{22}$ nanoscale thermometry and sensing. ${ }^{6}$ Accordingly, the efficient upconversion photoemission has been extensively investigated in various luminescent systems such as organic dyes, quantum dots, nanobelts, carbon nanotubes and especially lanthanide-doped upconversion nanoparticles. ${ }^{23-26}$

The efficiency of phonon-assisted upconversion can be enhanced by a large optical transition strength of emitters, or by realizing resonant conditions, that is, incident or/and emitted photon energy matches the resonance level of material system. Owing to the reduced dielectric screening and enhanced Coulomb attraction, the electron-hole pairs formed in monolayer semiconductors have a Bohr radius of $\sim 1 \mathrm{~nm}$ and a binding energy of $500 \mathrm{meV}$ (over an order of magnitude larger than conventional semiconductors), thus the fundamental optoelectronic properties are determined by excitonic effects at both cryogenic and room temperatures. ${ }^{27-34}$ The optical transition strength and phonon-exciton interaction effects are strongly enhanced in comparison to traditional 3D and quasi2D semiconductors. ${ }^{33,35}$ Hence the monolayer semiconductors can provide a great platform for the fundamental studies and applications of efficient phonon-assisted upconversion, ${ }^{35,36}$ such as excitonic upconversion lasing, optical refrigeration of excitonic devices. However, the research on the further enhancement of upconverted emission at low-threshold excitation intensity for 2D excitons is still in an early stage.

Coupling the quantum emitters to an optical cavity can significantly change the interaction between the emitter and its local optical environment. ${ }^{37,38}$ Moreover, the resonance frequencies of localized surface plasmons (LSPs) can be conveniently tailored by changing the size, shape, and interparticle separation. Here, we report the observation of the enhanced upconverted emission of two-dimensional excitons in doubly resonant plasmonic nanocavities. By integrating monolayer $\mathrm{WSe}_{2}$ into designed plasmonic nanocavities that doubly resonated with the incident or/and emitted photons, the deep subwavelength mode volume of cavity resonances can provide locally enhanced electromagnetic (EM) fields, and thereby enhance the phonon mediated optical absorption. Additionally, the spontaneous emission rate of the emitter can be accelerated via Purcell factor in weak coupling regime, leading to the plasmon-enhanced luminescence. ${ }^{39-42}$ Eventually, the upconverted emission amplification of $>1000$ folds and the decrease of $2 \sim 3$ orders of magnitude for saturated excitation energy density are achieved. Therefore, the plasmonic nanocavity is a promising method to realize the enhancement of upconverted emission at low-threshold excitation intensity for 2D excitons.

\section{Results and discussion}

Design and characterization of plasmonic upconverter devices. To enhance excitonic unconverted emission in doubly resonant plasmonic nanocavities, the nanoparticle-on-mirror geometry (NPoM, 
equivalent to gap-mode patch antennas) was adopted, placing mechanically exfoliated monolayer $\mathrm{WSe}_{2}$ in the gap between nanoparticles and a mirror underneath. ${ }^{38,42}$ Figure 1a illustrates the schematic of the designed $\mathrm{Au}$ nanocube $(\mathrm{AuNC}) / \mathrm{WSe}_{2} / \mathrm{substrate}$ plasmonic upconverter devices, where the substrate consists of a $5 \mathrm{~nm} \mathrm{Al} 2 \mathrm{O}_{3}$ spacer and a $50 \mathrm{~nm}$ Au layer evaporated on a $\mathrm{Si} / \mathrm{SiO}_{2}$ wafer. The desired band alignment diagram of monolayer $\mathrm{WSe}_{2}$ embedded in plasmonic nanocavities is depicted in Figure 1b, where the photon upconversion process of 2D excitons is also sketched. In monolayer WSe2, the electrons at ground state are excited and relaxed as excitons by absorbing a photon and phonons simultaneously (red arrow), where the photon energy $\hbar \omega_{1}$ locates at the long-wavelength tail of the absorption spectrum. Then the formed excitons can recombine via spontaneous emission of an upconverted photon with energy $\hbar \omega_{2}>\hbar \omega_{1}$ (yellow arrow). For the elaborate plasmonic nanocavities, the two cavity modes doubly resonant with the incident and emitted photon energy to guarantee both the excitation and emission processes are enhanced.

a

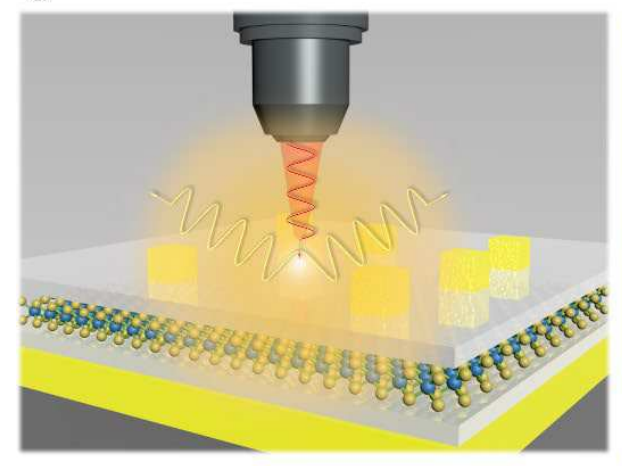

b

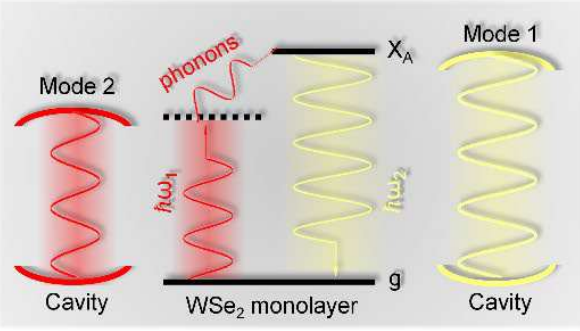

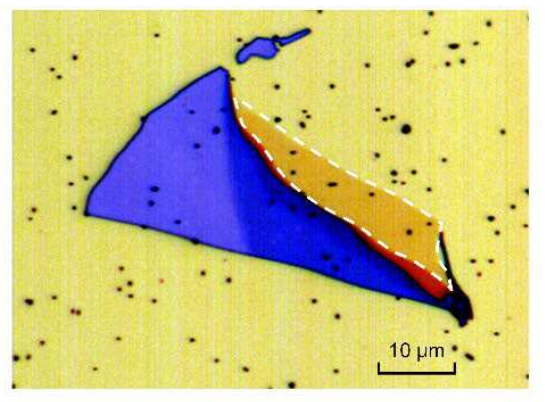

d

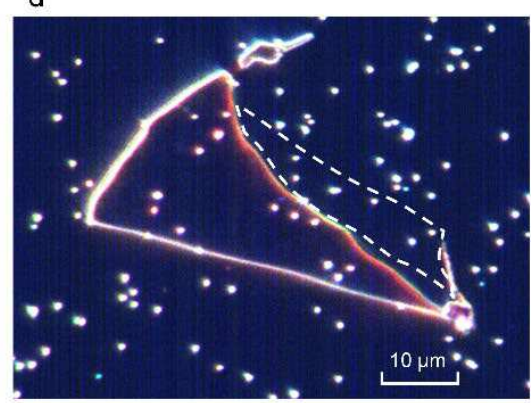

e
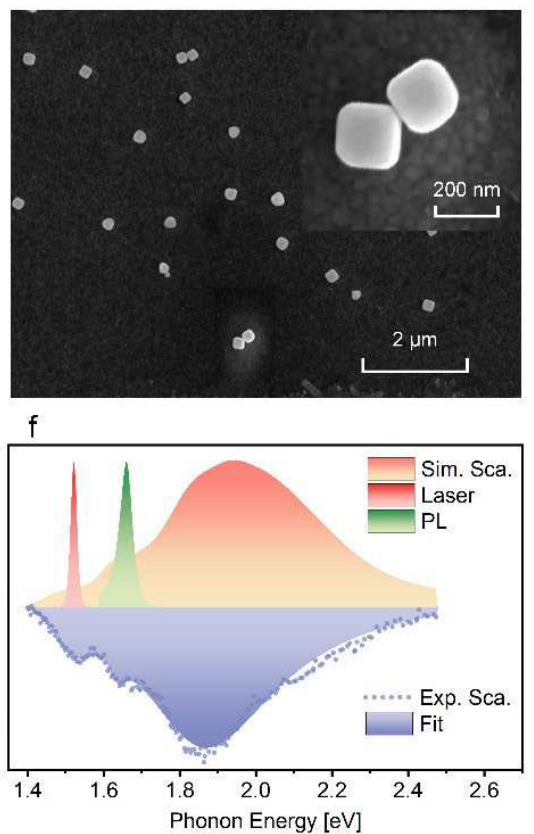

Figure 1. Design and characterization of plasmonic upconverter devices. (a) Schematic of the designed Au nanocube/WSe 2 /substrate plasmonic upconverter devices. (b) Desired band alignment diagram of monolayer WSe $\mathrm{W}_{2}$ in plasmonic nanocavities and the photon upconversion process of 2D excitons. (c) Bright- and (d) dark-field microscope optical images of a representative sample. (e) Scanning electron micrograph of $170 \mathrm{~nm}$ Au nanocube on substrate, and the inset shows the zoom-in image from the top view. (f) Excitation laser and monolayer WSe $\mathrm{PL}_{2}$ spectra overlap with simulated scattering spectrum of a plasmonic nanocavity, which is consistent with experimental results. The excitation laser and upconverted emission spectra doubly resonant with plasmonic cavity modes at $\lambda \approx 1.52 \mathrm{eV}$ and $1.67 \mathrm{eV}$, respectively.

Figures 1c and 1d show bright- and dark-field optical micrograph of a representative sample, respectively. The exfoliated monolayer $\mathrm{WSe}_{2}$ was transferred on the $5 \mathrm{~nm} \mathrm{Al} \mathrm{O}_{3} / 50 \mathrm{~nm} \mathrm{Au} / \mathrm{SiO}_{2} / \mathrm{Si}$ substrate fabricated by evaporation coating. Then AuNCs were sparsely deposited onto the monolayer 
WSe2 flake by drop casting. The separation between the AuNCs and monolayer $\mathrm{WSe}_{2}$ is the organic adhesive layer with a thickness of $\sim 1 \mathrm{~nm}$. AuNCs were synthesized using the seed mediated method, and the averaging side length of $\sim 170 \mathrm{~nm}$ can be obtained from the SEM image depicted in Figure 1e. The spacer layers among Au film, WSe 2 , and AuNCs can prevent hot carriers that decay from LSPs or surface plasmon polaritons (SPPs) injecting into the monolayer $\mathrm{WSe}_{2}$ (see supporting $\mathrm{S} 1$ for more details).

Figure $1 \mathrm{f}$ presents the simulated and experimental scattering spectra to confirm the optimal matching among excitation laser, upconversion photons and cavity modes. Noticeably, the simulated spectrum is consistent with experimental results. The spectra of excitation laser and monolayer WSe2 photoluminescence (PL) overlap with simulated scattering spectrum of a plasmonic nanocavity. It means that the excitation laser and upconverted emission spectra are doubly resonant with plasmonic cavity modes at $\lambda \approx 1.52 \mathrm{eV}$ and $1.67 \mathrm{eV}$, respectively. The prominent peak of upconverted emission spectrum can be attributed to the neutral exciton $(\mathrm{X})$ in monolayer WSe2. Clearly, the emitted photon energy is $150 \mathrm{meV}$ higher than the incident photons, which is five times larger than the results in ref. [34]. The photon energy difference $\Delta \mathrm{E}$ is much larger than the intrinsic phonon energy of $\sim 30 \mathrm{meV}$ in monolayer $\mathrm{WSe}_{2}$ (see supporting S2 for more details), which implies that multiphonon are involved in the excitonic upconverted emission.

Phonon-assisted excitonic upconverted emission of monolayer WSe 2 . As depicted in Figure $1 \mathrm{~b}$, the excitonic unconverted emission is determined by incident photons and the phonons in monolayer WSe2. Figure 2a shows the incident photon energy-dependent upconverted emission spectrum, where the excitation photon energy ranging from $1.522 \mathrm{eV}$ to $1.534 \mathrm{eV}$ were precisely controlled by wavelengthtunable femtosecond pulse oscillator. As the excitation photon energy gradually overlaps with the exciton emission peak, the spectral shape and peak position of unconverted emission is permanent, whereas the intensity increase evidently. To quantify the dependence of total unconverted emission intensity on excitation photon energy, the integrated intensity of upconversion spectra (Figure 2a) as a function of the energy difference $\Delta \mathrm{E}$ between the excitonic emissions and the excitation photons is presented in Figure 2b. The experimental results can be well fitted by the excitons obeyed classical Boltzmann function.

As the quanta of the crystal vibrational field, phonon energy and density are determined by lattice temperature. Additionally, the energy gap related excitonic emission peak of semiconductors also depends on temperature, considering the temperature-dependent lattice dilatation and electron-lattice interaction. ${ }^{43}$ Therefore, we can expect that the temperature plays a critical role on the spectral shape, intensity and peak position of excitonic upconverted emission. Figure 2c presents the temperaturedependent excitonic upconverted emission spectra. It can be clearly observed the exciton peak 
broadening and red shift with rising temperature, which is consistent with the exciton PL spectra under standard excitation. The broadening and red shift of spectra can be well explained by the well-known Varshni equation and the interaction of excitons with the longitudinal-acoustical (LA) and longitudinal-optical (LO) phonon modes of lattice (Figure S3). Figure 2d depicts the temperaturedependent unconverted emission intensity. With the temperature reduces, the energy difference $\Delta \mathrm{E}$ between the excitonic emissions and the exciting photons increase gradually due to the blue shift of exciton peak, and the phonons density decrease. Thus the total unconverted emission intensity drop dramatically so that the detectable upconverted emission (above the noise floor) can't be found for the temperature lower than $250 \mathrm{~K}$. Considering multiphonon are involved in the unconverted process, the experimental results in Figure $2 \mathrm{~b}$ are proportional to $\bar{n}^{x}$, where $\bar{n}=1 /\left[\exp \left(\hbar \omega_{q} / k_{B} T\right)-1\right]$ is the temperature-dependent average population of phonons gas in monolayer $\mathrm{WSe}_{2}$, and $x$ is the involved phonon number in single unconverted emission. The green and red dotted lines show the fitted results for $x=3$ and 5, respectively. It illustrates that the higher temperature, the less phonons needed in single unconverted emission, which is consistent with the reduced $\Delta \mathrm{E}$ with rising temperature (Figure $2 \mathrm{c}$ ). In conclusion, the excitation photon energy- and temperature-dependent measurements show behavior consistent with the physical scenario depicted in Figure 1b, thereby provide an undoubted evidence for phonon-assisted excitonic upconverted emission.

a

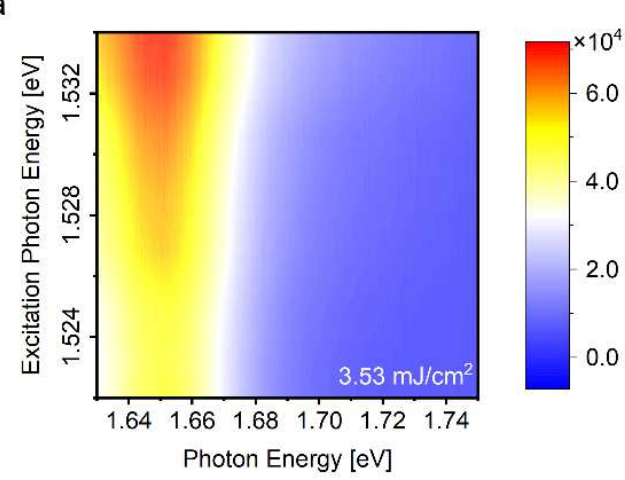

b

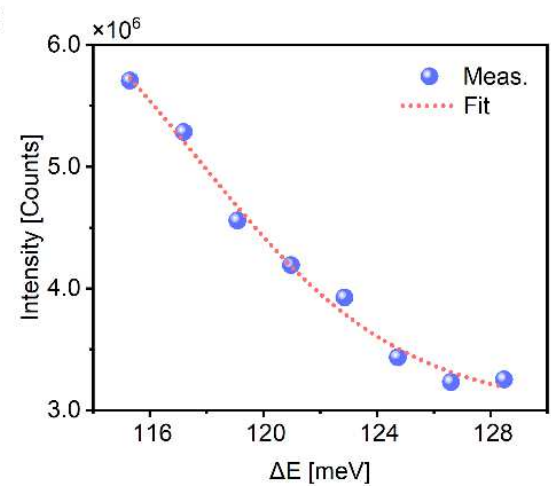

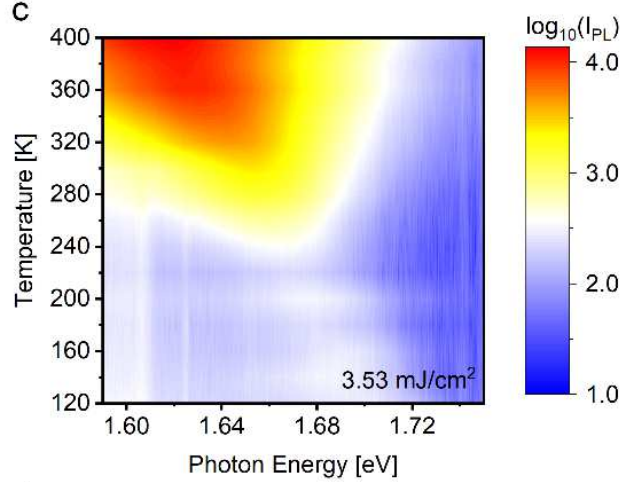

d

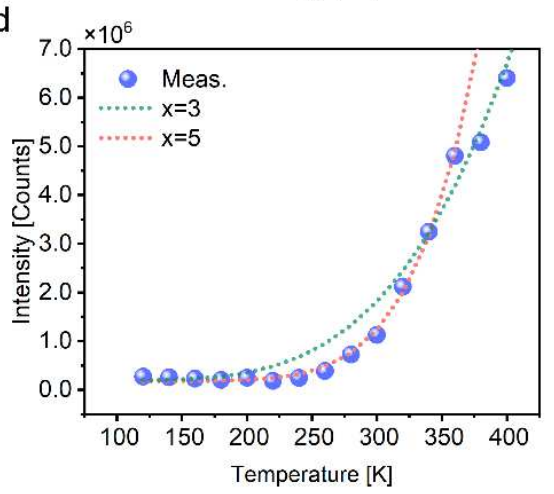

Figure 2. Phonon-assisted excitonic upconverted emission of monolayer $\mathrm{WSe}_{2}$. (a) Excitation photon energydependent PL spectra. (b) Excitation photon energy-dependent integrated PL intensity calculated from (a). (c) Temperature-dependent PL spectra for excitation photon energy at $1.52 \mathrm{eV}$. (d) Temperature-dependent integrated PL intensity calculated from (c). 
147 Enhanced excitonic unconverted emission in plasmonic cavity. To explore the enhancement of excitonic unconverted emission in plasmonic cavity, we performed excitation energy densitydependent measurements with the excitation photon energy of $1.52 \mathrm{eV}$ at room temperature and ambient condition. Firstly, the experiments were carried out on the monolayer $\mathrm{WSe}_{2}$ transferred on $\mathrm{SiO}_{2} / \mathrm{Si}$. Figure 3a presents the excitation energy density-dependent unconverted emission map. As the excitation energy density increases, the intensity increases evidently, whereas the spectral shape and peak position of unconverted emission is permanent, illustrating that the thermal and renormalization effects can be reasonably neglected at such excitation power level. Meanwhile, the dependence of total unconverted emission intensity on excitation energy density is shown in Figure 3d, which behaves as an obvious linear relation. The possibility of nonlinear optical generation of the observed PL upconversion, such as two-photon excitation-induced emission ${ }^{44,45}$ and exciton Auger scattering ${ }^{46,47}$ can be ruled out, that is, the phonon-assisted excitonic upconverted emission is further confirmed.
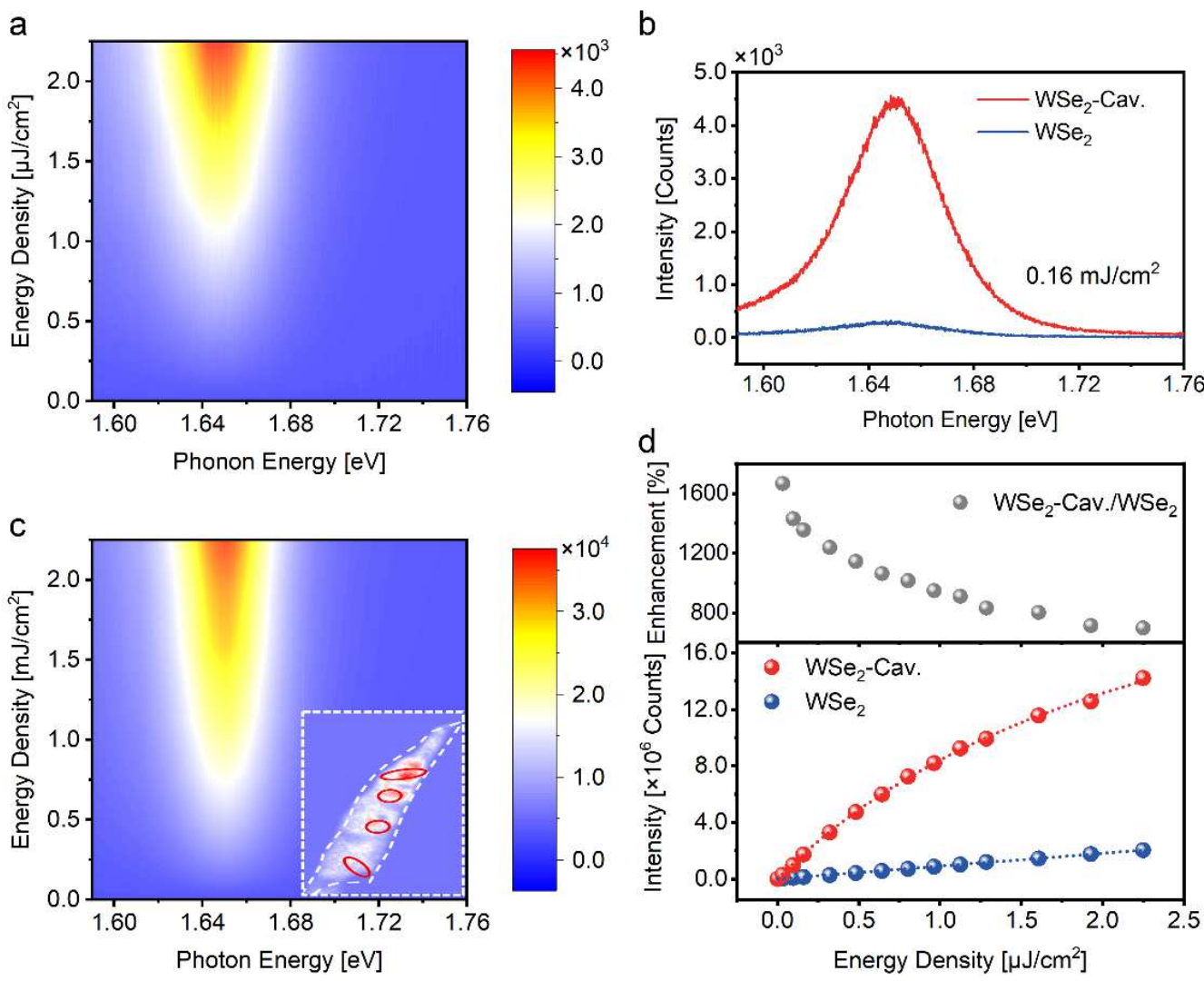

Figure 3. Upconversion amplificated by plasmonic cavity. (a) Excitation energy density-dependent unconverted PL spectra for monolayer $\mathrm{WSe}_{2}$ on $\mathrm{SiO}_{2} / \mathrm{Si}$. (b) Excitation energy density-dependent unconverted PL spectra for monolayer $\mathrm{WSe}_{2}$ in designed plasmonic cavity. (c) Enhanced unconverted PL spectra of monolayer $\mathrm{WSe}_{2}$ in designed plasmonic cavity at the excitation energy density of $0.16 \mathrm{~mJ} / \mathrm{cm}^{2}$. Inset: PL maps of the plasmonic nanocavity effect on exciton emission, where the nanocubes and the monolayer $\mathrm{WSe}_{2}$ are marked by red solid line and white dashed line. (d) Exitation energy density-dependent integrated unconverted PL intensity (top) and the enhancement (down) for monolayer $\mathrm{WSe}_{2}$ in designed plasmonic cavity. 
In well-designed plasmonic upconverter devices (Figures 1a and 1f), the excitation laser and upconverted emission can be expected, comparing with monolayer $\mathrm{WSe}_{2}$ on $\mathrm{SiO}_{2} / \mathrm{Si}$ under the same conditions. Figure $3 \mathrm{~b}$ shows the PL spectra of $\mathrm{WSe}_{2}$ on $\mathrm{SiO}_{2} / \mathrm{Si}$ and plasmonic cavity at the excitation energy density of $0.16 \mathrm{~mJ} / \mathrm{cm}^{2}$, where a 13.5 folds enhancement is obtained. To explicitly reveal the dependence of the enhancement on excitation energy density, the excitation energy density-dependent unconverted emission map for the $\mathrm{WSe}_{2}$ in plasmonic cavity was measured and plotted in Figure 3c. The spectral shape and peak position remain nearly unchanged, whereas the relationship between upconversion intensity and excitation energy density varies significantly, that is, the upconverted emission gradually increases to saturation and the enhancement accordingly reduces with the increase of excitation energy density, as shown in Figure 3d. The saturation phenomenon in unconverted process could be ascribed to the saturated absorption and the exciton radiative lifetime. Additionally, the inset of Figure 3c shows PL maps of the plasmonic nanocavity effect on exciton emission excited by the photon energy of $3.04 \mathrm{eV}$. The nanocubes and the monolayer $\mathrm{WSe}_{2}$ are marked by red solid line and white dashed line, respectively. Very noteworthy here is that the monolayer $\mathrm{WSe}_{2}$ in nanocavity exhibits a bright luminescence while the weaker emission was found in the absence of nanocubes.

In light of the fact that only the region close to the nanocube rather than the total excitation region is enhanced, the real upconversion enhancement in the plasmonic cavity should be much larger than the value presented in Figure 3d. Commonly, to quantify the actual upconversion enhancement in the plasmonic cavity, the PL enhancement factor can be defined as ${ }^{40}$

$$
\langle E F\rangle=\frac{I_{P C}-I_{0}}{I_{0}} \frac{S_{0}}{S_{P C}}
$$

where $I_{P C}$ is the total upconversion intensity of the monolayer $\mathrm{WSe}_{2}$ in plasmonic cavity, $I_{0}$ is upconversion intensity of the monolayer $\mathrm{WSe}_{2}, S_{0}$ defines the excitation area in our measurements $\left(1.84 \mu \mathrm{m}^{2}\right)$, while $S_{P C}$ represents the hotspot area in plasmonic cavity that enhances the upconversion of monolayer $\mathrm{WSe}_{2}$. For brevity, we assume $S_{P C}$ to be the area that monolayer $\mathrm{WSe}_{2}$ contacts with plasmonic cavity $\left(0.0289 \mu \mathrm{m}^{2}\right)$. The range of $400 \sim 1100$ folds enhancement can be achieved corresponding to Figure 3d.

In addition, the dependence of upconversion intensity on excitation energy density for plasmonic upconverter devices in Figure 3d can be well fitted by

$$
I=I_{\text {sat }} \frac{f}{f+f_{\text {sat }}}
$$

197 where $I$ and $I_{\text {sat }}$ are total upconverted emission intensity and the corresponding saturation value, $f$ and $f_{\text {sat }}$ are the excitation energy density and the corresponding saturation value, respectively. The optimal 
199 fitting parameters of red dotted curve are $I_{\text {sat }}=3.3 \times 10^{7}$ counts and $f_{\text {sat }}=2.56 \mathrm{~mJ} / \mathrm{cm}^{2}$. For the excitation energy density $f<<f_{\text {sat }}$, the equation (2) can be simplified as $I=I_{\text {sat }} f / f_{\text {sat }}$. Based on the fitted slope of the blue dotted line in Figure 3d, the saturated excitation energy density can be estimated as $14.41 \mathrm{~mJ} / \mathrm{cm}^{2}$, assuming the $I_{\text {sat }}=1.4 \times 10^{7}$ counts for monolayer $\mathrm{WSe}_{2}$ transferred on $\mathrm{SiO}_{2} / \mathrm{Si}$ (Figure S4). Actually, the measured energy density-dependent emission intensity (red points) collected the upconverted emission in both plasmonic cavity and neighbouring free space. The real saturated excitation energy density in our designed doubly resonant plasmonic cavity can be estimated as 32.9 $\mu \mathrm{J} / \mathrm{cm}^{2}$ (see Supporting S5 for more details), which is reduced by $2 \sim 3$ orders of magnitude compared with free space.

Mechanism of enhanced excitonic unconverted emission in plasmonic cavity. In the doubly resonant plasmonic cavity, the upconverted emission enhancement originates from three processes: enhanced light collection, enhanced excitation rate and quantum efficiency enhancement arising from Purcell effect, resulting in an average upconversion enhancement factor of $400 \sim 1100$ folds. Firstly, as a nanoscale patch antenna, the plasmonic cavity can improve the directionality of emission and thereby enhance the light collection for an optical system with fixed numerical aperture (NA) (Figure 4a). The radiation pattern of the antenna can be simulated by 3D-FDTD simulations (See supporting S6 and S7 for more details). As shown in Figure 4b, for the in-plane dipole source with center wavelength of $750 \mathrm{~nm}$ and spectral width of $25 \mathrm{~nm}$ (similar with the PL Spectra of monolayer WSe2), the far-field radiation pattern has a single lobe oriented in the surface-normal direction. The fraction of emitted light collected by the objective lens $\mathrm{NA}=0.5$ adopted in our measurments can be calculated to be $44.4 \%$, which is $\sim 1.7$-folds higher than that of the monolayer $\mathrm{WSe}_{2}$ transferred on $\mathrm{Si} / \mathrm{SiO}_{2}$.

Besides, to clarify how plasmonic cavity observably molds light field and enhance upconverted emission, the 3D-FDTD simulations were performed to simulate the charge and field distribution around plasmonic cavity. Figures $4 \mathrm{~d}$ and $4 \mathrm{~g}$ show the charge distributions at the monolayer WSe 2 , corresponding to the resonant energy at $1.67 \mathrm{eV}$ and $1.52 \mathrm{eV}$ in far-field scattering spectrum (Figure 1f), respectively. The results reveal that the resonance at $1.67 \mathrm{eV}$ can be ascribed to the mixed dipolar modes, whereas the resonance at $1.52 \mathrm{eV}$ is arising from the coupled quadripolar mode. Figures $4 \mathrm{e}$ and 4h show the field distribution at $x z$ plane of two plasmonic cavity modes, as well as Figures $4 \mathrm{f}$ and $4 \mathrm{i}$ present the field distribution $\left(\left|\mathrm{E} / \mathrm{E}_{0}\right|\right)$ at the monolayer $\mathrm{WSe}_{2}$ (See Figures S8 and S9 for more details). These filed distributions demonstrate that the plasmonic modes at $1.67 \mathrm{eV}$ and $1.52 \mathrm{eV}$ confine the excitation light filed in the gap with a maximum field enhancement $\left(\left|\mathrm{E} / \mathrm{E}_{0}\right|\right)$ up to 40 . The maximum enhancement of light intensity $\left(\left|\mathrm{E}_{\mathrm{E}} / \mathrm{E}_{0}\right|^{2}\right)$ of $>1600$-folds for the mode at excitation photon energy $(1.52$ $\mathrm{eV}$ ) can provide a reasonable explanation for the 2 3 orders decrease of saturated excitation energy density in the plasmonic cavity. 
a

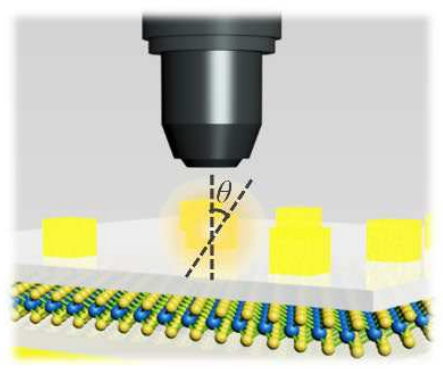

d

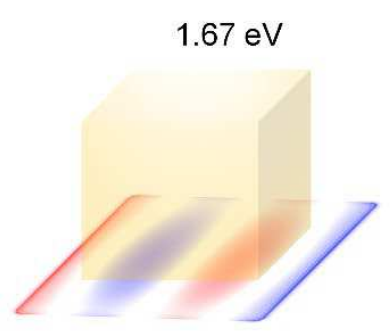

g

$1.52 \mathrm{eV}$

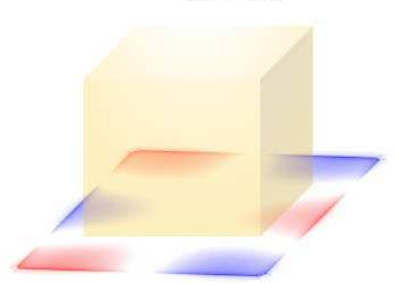

b

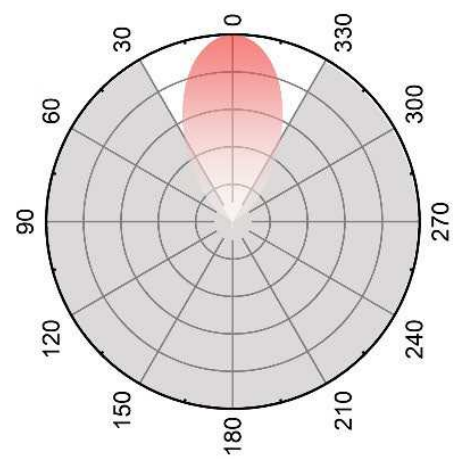

C

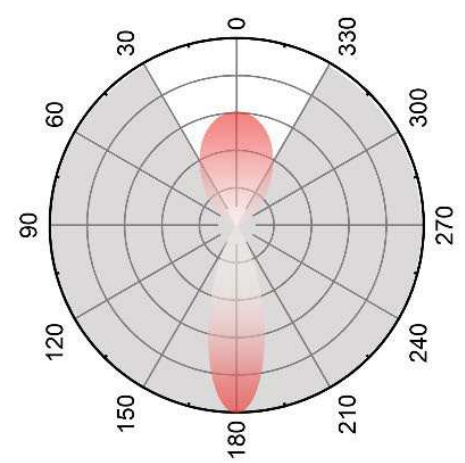

e

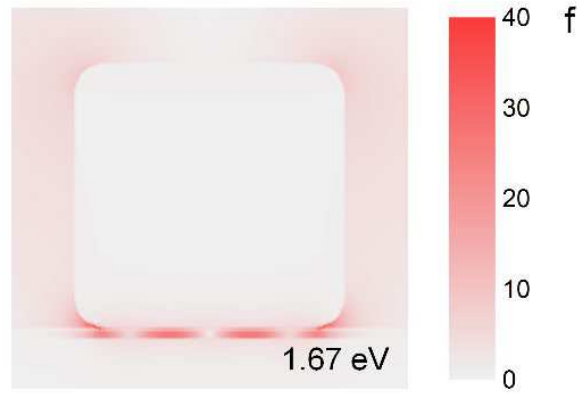

h

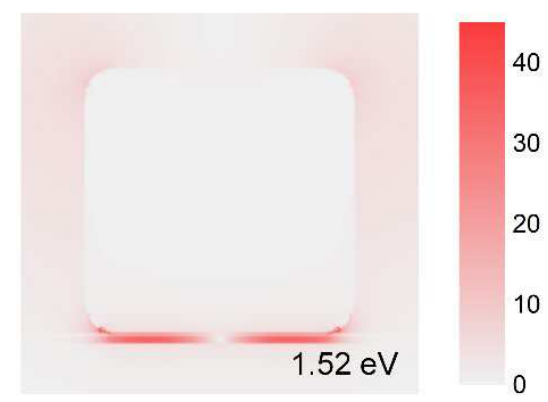

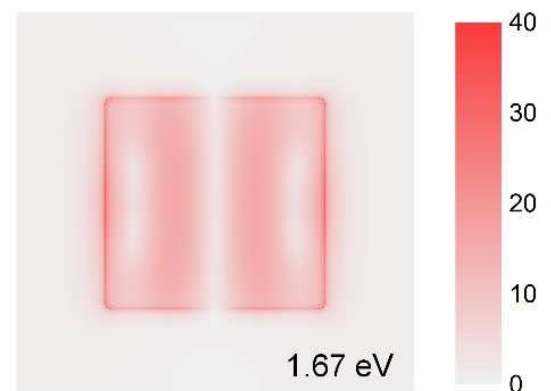

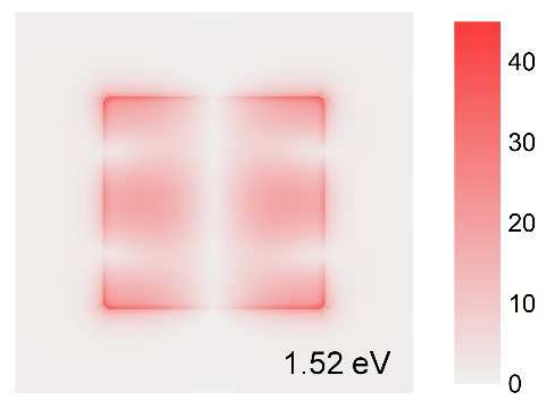

Figure 4. Mechanism of the enhanced upconversion of plasmonic cavity. (a) Schematic of the setup for collecting upconverted emissions in our experiments. (b), (c) Far-field angular radiation patterns for monolayer $\mathrm{WSe}_{2}$ in plasmonic cavity (b) and free space(c). (d), (e), (f) Charge and field distribution $\left(\left|\mathrm{E} / \mathrm{E}_{0}\right|\right)$ around plasmonic cavity for the mode at emitted photon energy $(1.67 \mathrm{eV})$. (g), (h), (i) Charge and field distribution $\left(\left|\mathrm{E} / \mathrm{E}_{0}\right|\right)$ around plasmonic cavity for the mode at excitation photon energy $(1.52 \mathrm{eV})$.

Additionally, field enhancements up to 40 folds for the modes at the PL energy (1.52 eV) of A exciton can yield an appreciable Purcell effect. The quantum efficiency from exciton to upconverted emission can be enhanced by the Purcell effect, which can boost the rate of spontaneous emission by manipulating the local density of optical states according to Fermi's golden rule. In the resonant cavity, the local density of states can be greatly increased, and the enhancement of spontaneous emission rate can be expressed as Purcell factor ${ }^{48}$

$$
F=\frac{\gamma_{\text {cav }}}{\gamma_{0}}=\frac{3}{4 \pi^{2}} \frac{Q}{V_{\text {mode }}}\left(\frac{\lambda}{n}\right)^{3}
$$

where $\gamma_{\mathrm{cav}}$ and $\gamma_{0}$ are the spontaneous emission rate of the emitter in cavity and free space, respectively, 
$247 Q$ is the cavity quality factor, $V_{\text {mode }}$ is the mode volume, $\lambda$ is the resonant wavelength, $n$ is the refractive 248 index of the medium. It illustrates that the plasmonic nanocavity can possesses a large Purcell factor 249 owing to the nanoscopic mode volume for a modest cavity quality factor. Thus the 2D excitons in the 250 elaborate plasmonic cavity can exhibit a giant enhancement of spontaneous emission rate and 251 luminescence.

252 Purcell effect modulated exciton relaxation in plasmonic cavity. To gain more insight into the 253 Purcell-enhanced upconverted emission, the exciton relaxation dynamics in the plasmonic cavity and 254 free space were measured by femtosecond pump-probe spectroscopy. In the measurements, to explore 255 the relaxation dynamics of the A excitons in monolayer $\mathrm{WSe}_{2}$, the photon energy of pump and probe 256 pulses were chosen as $3.04 \mathrm{eV}$ and $1.65 \mathrm{eV}$, and the pump and probe energy density were $12.9 \mu \mathrm{J} / \mathrm{cm}^{2}$ and $1.2 \mu \mathrm{J} / \mathrm{cm}^{2}$, respectively.
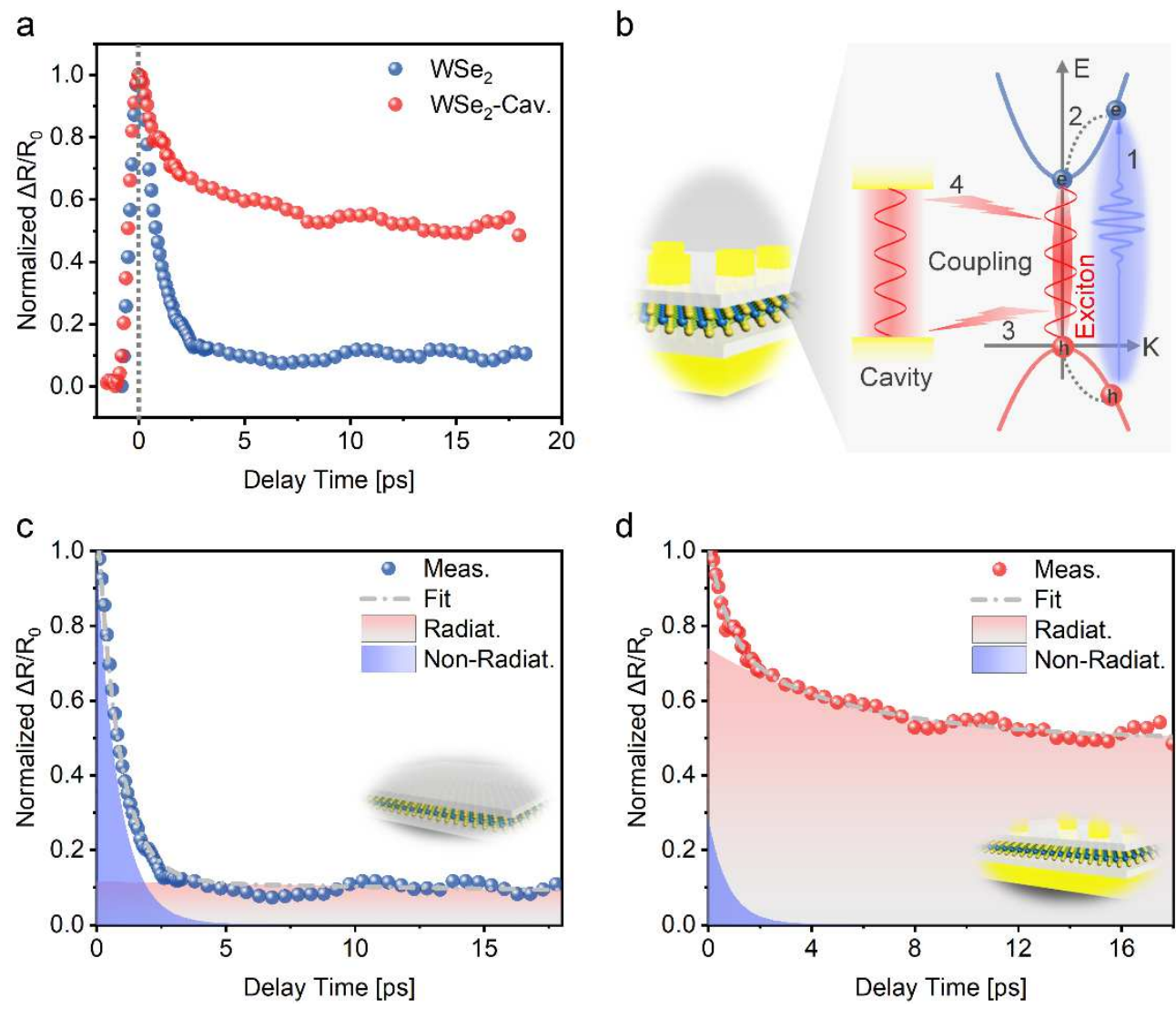

Figure 5. Purcell effect modulated exciton relaxation in plasmonic cavity. (a) Normalized differential reflection signal $\Delta R / R_{0}$ for the monolayer $\mathrm{WSe}_{2}$ in free space and plasmonic cavity. (b) Schematic diagram of purcell effect modulated exciton relaxation in plasmonic cavity: 1. Nonresonant excitation, 2. Intraband relaxation of energetic carriers, 3-4. Coupling between cavity and exciton emission. (c), (d) Fittings of the exciton relaxation in free space (c) and plasmonic cavity (d), respectively.

Figure $5 \mathrm{a}$ shows the normalized differential reflection signal $\Delta R / R 0$ for the monolayer $\mathrm{WSe}_{2}$ on 
relaxation process in plasmonic cavity changes dramatically due to the Purcell effect arising from the resonance between A exciton peak and cavity mode. The schematic diagram of Purcell effect modulated exciton relaxation in plasmonic cavity is sketched in Figure 5b. The energetic electrons and holes are excited by pump pulse (1) and then relax to excitons (2). These high density excitons either recombine radiatively with the emission of photons (3) or dissipate non-radiatively through manybody scatterings including multi-exciton annihilation and exciton-exciton annihilation. ${ }^{49,50}$ In the resonant plasmonic cavity, the coupling between cavity and exciton emission ( 3 and 4 ) can prominently improve the spontaneous emission rate of exciton through Purcell effect, thereby reconstruct competitive relationship between radiative recombination and nonradiative annihilation during the relaxation of excitons.

Generally, considering multi-exciton annihilation $\left(\sim N^{x}, x>2\right)$, exciton-exciton annihilation $\left(\sim N^{2}\right)$ and exciton radiative recombination $(\sim N)$, the rate equation that describes the exciton relaxation dynamics can be written as following

$$
\frac{\partial N}{\partial t}=-\alpha N^{4}-\beta N^{2}-\gamma N
$$

where $N$ is exciton density, $\alpha$ and $\beta$ are biexciton-biexciton annihilation rate and exciton-exciton annihilation rate, and $\gamma$ is exciton decay rate of single exciton radiative recombination. Comparing with radiative recombination, the many body scattering $\left(\sim N^{x}, x \geqslant 2\right)$ is a much faster process. Therefore, to quantify the time constants of fast nonradiative annihilation and slow radiative recombination, the transient decay dynamics can be approximately fitted into two exponential decay components.

Figures $5 \mathrm{c}$ and $5 \mathrm{~d}$ show the fitted exciton relaxation of the monolayer $\mathrm{WSe}_{2}$ in free space and plasmonic cavity. The monolayer $\mathrm{WSe}_{2}$ in free space (Figure 5c) undergoes the exciton relaxation with a fast decay time constant of 0.86 ps and a slow decay time constant of 87.47 ps, while the fast and slow decay time constants are $0.78 \mathrm{ps}$ and $5.66 \mathrm{ps}$ for the monolayer $\mathrm{WSe}_{2}$ in plasmonic cavity (Figure 5d), respectively (see Figure S10 for the monolayer $\mathrm{WSe}_{2}$ on $\mathrm{Au}$ film). That indicates a 15-fold radiative decay rate enhancement, the actual enhancement can be much greater considering the collected signals are comprised of the excitons coupled to plasmonic cavity and neighbouring excitons without coupling. Accordingly, the constituents of radiative recombination (filled with red color) and nonradiative annihilation (filled with blue color) in exciton relaxation were significantly altered, that is, the nonradiative annihilation is suppressed and the radiative recombination is greatly enhanced in plasmonic cavity, which agrees with the enhancement of upconverted emission in Figure 3.

\section{Conclusion}

In summary, the multiphoton upconverted emission of 2D excitons can be greatly enhanced by the elaborate doubly resonant plasmonic nanocavity. The upconverted emission amplification of $>1000$ 
folds and the decrease of nearly three orders of magnitude for saturated excitation energy density has been achieved. It can be attributed to the enhanced light collection, enhanced excitation rate and quantum efficiency enhancement arising from Purcell effect. These findings pave the way to the development of excitonic upconversion lasing, nanoscopic thermometry and sensing, and open up the possibility of optical refrigeration in future 2D electronic or excitonic based devices.

\section{Methods}

Sample preparations. Firstly, a $50 \mathrm{~nm}$ Au film and a $6 \mathrm{~nm} \mathrm{Al}_{2} \mathrm{O}_{3}$ isolation layer were deposited on the surface of silicon wafers with $300 \mathrm{~nm} \mathrm{SiO}_{2}$ by electron beam evaporation, then an exfoliated $\mathrm{WSe}_{2}$ monolayer was transferred onto the substrate by polydimethylsiloxane (PDMS) assisted dry method. In the following process, the Au nanocubes (AuNCs) with size of $170 \mathrm{~nm}$ were uniformly distributed on the surface of the sample by chemical-assisted method. In a typical process, $58.6 \mathrm{mg} \mathrm{NaCl}$ and $212.3 \mathrm{mg}$ poly(sodium-p-styrenesulfonate) (PSS) were dissolved in $1 \mathrm{ml}$ deionized (DI) water to obtain PSS solutions. The substrate containing the sample was then fully immersed in PSS solutions at room temperature and held for 5 minutes, after which the substrate was carefully cleaned with DI water. The purpose of this treatment is to prevent AuNCs from coalescing on the substrate surface during the following process. ${ }^{51}$ A pipette gun is then used to drop $50 \mathrm{mg} / 1$ of AuNCs DI water solutions onto the substrate in an amount of about 100 microlitres per square centimetre. Finally, the substrate is steamed to dry the solvent on a hot plate at $90{ }^{\circ} \mathrm{C}$ in air.

Dark-field scattering Measurements. The dark-field scattering mapping and spectra were measured with a commercial hyperspectral imaging system (Cytoviva, HISV3). The white light was focused by a $100 \times$ objective with a high numerical aperture (Olympus, MPlanFLN, NA $=0.9$ ). The mapping of scattering signal was realized with a precisemotorized transla- tion stage, and spectral profiles of all pixels could be obtained. Scattering signals were recorded by the spectrometer (Horiba, iHR550) cooled to $-60{ }^{\circ} \mathrm{C}$. Scattering spectra of samples were corrected with the substrate, using the build-in software (Cytoviva, ENVI 4.8).

Confocal PL maps measurements. PL maps were performed by ISS Q2 confocal laser scanning system coupled to a Nikon TE2000 microscope with the $60 \times / 1.2$ NA WI objective lens. The excitation wavelength is $405 \mathrm{~nm}$ (5000 Hz repetition rate), PL emission signals were collected through a $480 \mathrm{~nm}$ long-pass edge filter.

Mirco PL Spectra Measurements. The femtosecond pulses $(817 \mathrm{~nm}, 73 \mathrm{fs}, 80 \mathrm{MHz})$ emitted from mode-locked oscillator (Tsunami 3941C-25XP) were focused by an infinity-corrected long work distance microobjective (Mitutoyo, $100 \times, \mathrm{NA}=0.5$ ) to excite sample. The excitation sites was confirmed by an EMCCD camera (Andor Ixon 888) with the micro objective and matched widefield 
tube lens (Thorlabs TTL200-A). For spectral measurements, the $800 \mathrm{~nm}$ short-pass edge filter (Thorlabs FELH0650) was used to block excitation light. The filtered light was coupled to a spectrometer (Acton SP2500) equipped with a liquid nitrogen cooled CCD.

Ultrafast measurements. Ultrafast pump probe measurements in reflection configuration were carried out. The femtosecond pulses $(817 \mathrm{~nm}, 73 \mathrm{fs}, 80 \mathrm{MHz})$ were split into two parts. One of them passed through a $\mathrm{BBO}$ crystal to produce the $408 \mathrm{~nm}$ pump pulses, while the other one was focused into a photonic crystal fiber (Newport SCG-800) to generate the super-continuum white light. The probe pulses were then selected with a $750 \pm 10 \mathrm{~nm}$ (Thorlabs FB750-10) bandpass filters. The spot size of the focused probe and pump laser was $<1 \mu \mathrm{m}$. The delay time between pump and probe pulses was controlled by a steeper linear stage (Newport M-ILS150PP). To improve the signal-to-noise ratio, the reflected probe pulses passed through a $650 \mathrm{~nm}$ longpass edge filter (Thorlabs FEL0650) and then were detected by a high-sensitivity photomultiplier (Thorlabs PMM02) connected with the phase lockin amplifier (Stanford SR830).

Finite-Difference Time-Domain (FDTD) Simulations. 3D FDTD simulations were was employed to simulate electromagnetic field properties. In the simulation of scattering spectra, reflectance spectra, charge and electromagnetic field distributions, the total-field scattered-field (TFSF) source with the wavelength ranging from 500 to $900 \mathrm{~nm}$ was adopted. In addition, the in-plane dipole source arrays with center wavelength of $750 \mathrm{~nm}$ and spectral width of $25 \mathrm{~nm}$ were used to simulate the far-field angular radiation patterns. The perfectly matched layer (PML) was set as boundary conditions, and the mesh size around plasmonic cavity was $1 \mathrm{~nm}$. Relative permittivities of $\mathrm{Si}, \mathrm{SiO}_{2}, \mathrm{Al}_{2} \mathrm{O}_{3}, \mathrm{Au}$ and $\mathrm{WSe} 2$ monolayer were taken from literatures, ${ }^{52-54}$ and the thickness of $\mathrm{WSe}_{2}$ layer was set as $1 \mathrm{~nm}$.

\section{Data availability}

The data that support the findings of this study are available from the corresponding author upon request.

\section{References}

1 Wu, Y. et al. Upconversion superburst with sub-2 $\mu$ s lifetime. Nature Nanotechnology 14, 11101115, doi:10.1038/s41565-019-0560-5 (2019).

2 Chen, W. et al. Giant five-photon absorption from multidimensional core-shell halide perovskite colloidal nanocrystals. Nature Communications 8, 15198, doi:10.1038/ncomms15198 (2017).

$3 \mathrm{Wu}, \mathrm{M}$. et al. Solid-state infrared-to-visible upconversion sensitized by colloidal nanocrystals. Nature Photonics 10, 31-34, doi:10.1038/nphoton.2015.226 (2016). 
3674 Börjesson, K., Rudquist, P., Gray, V. \& Moth-Poulsen, K. Photon upconversion with directed emission. Nature Communications 7, 12689, doi:10.1038/ncomms12689 (2016).

5 Chen, C. K., de Castro, A. R. B. \& Shen, Y. R. Surface-Enhanced Second-Harmonic Generation. Physical Review Letters 46, 145-148, doi:10.1103/PhysRevLett.46.145 (1981).

6 Tran, T. T. et al. Anti-Stokes excitation of solid-state quantum emitters for nanoscale thermometry. Sci. Adv. 5, eaav9180 (2019).

7 Li, Y. et al. Giant two-photon absorption in monolayer MoS2. Laser \& Photonics Reviews 9, $427-$ 434, doi:https://doi.org/10.1002/lpor.201500052 (2015).

8 Hong, G. et al. Through-skull fluorescence imaging of the brain in a new near-infrared window. Nat. Photonics 8, 723-730, doi:10.1038/nphoton.2014.166 (2014).

9 Hong, G., Diao, S., Antaris, A. L. \& Dai, H. Carbon Nanomaterials for Biological Imaging and Nanomedicinal Therapy. Chemical Reviews 115, 10816-10906, doi:10.1021/acs.chemrev.5b00008 (2015).

10 Hong, G., Antaris, A. L. \& Dai, H. Near-infrared fluorophores for biomedical imaging. Nature Biomedical Engineering 1, 0010, doi:10.1038/s41551-016-0010 (2017).

11 Liu, Y. et al. Amplified stimulated emission in upconversion nanoparticles for super-resolution nanoscopy. Nature 543, 229-233, doi:10.1038/nature21366 (2017).

12 Fan, Y. et al. Lifetime-engineered NIR-II nanoparticles unlock multiplexed in vivo imaging. Nat. Nanotechnol. 13, 941-946, doi:10.1038/s41565-018-0221-0 (2018).

$13 \mathrm{Li}$, J. \& Pu, K. Development of organic semiconducting materials for deep-tissue optical imaging, phototherapy and photoactivation. Chem. Soc. Rev. 48, 38-71, doi:10.1039/C8CS00001H (2019).

14 Sun, Y. et al. Rhomboidal Pt(II) metallacycle-based NIR-II theranostic nanoprobe for tumor diagnosis and image-guided therapy. Proceedings of the National Academy of Sciences 116, 1968, doi:10.1073/pnas.1817021116(2019).

15 Zhou, H. et al. Upconversion NIR-II fluorophores for mitochondria-targeted cancer imaging and photothermal therapy. Nat. Commun. 11, 6183, doi:10.1038/s41467-020-19945-w (2020).

16 Deng, R. et al. Temporal full-colour tuning through non-steady-state upconversion. Nat. Nanotechnol. 10, 237-242, doi:10.1038/nnano.2014.317 (2015).

17 Chen, X. et al. Confining energy migration in upconversion nanoparticles towards deep ultraviolet lasing. Nat. Commun. 7, 10304, doi:10.1038/ncomms10304 (2016). 
18 Fernandez-Bravo, A. et al. Continuous-wave upconverting nanoparticle microlasers. Nat. Nanotechnol. 13, 572-577, doi:10.1038/s41565-018-0161-8 (2018).

19 Shang, Y. et al. Low threshold lasing emissions from a single upconversion nanocrystal. Nat. Commun. 11, 6156, doi:10.1038/s41467-020-19797-4 (2020).

20 Lamon, S., Wu, Y., Zhang, Q., Liu, X. \& Gu, M. Nanoscale optical writing through upconversion resonance energy transfer. Sci. $A d v .7$, eabe2209 (2021).

21 Shan, X. et al. Optical tweezers beyond refractive index mismatch using highly doped upconversion nanoparticles. Nat. Nanotechnol., 1-7 (2021).

22 Epstein, R. I., Buchwald, M. I., Edwards, B. C., Gosnell, T. R. \& Mungan, C. E. Observation of laser-induced fluorescent cooling of a solid. Nature 377, 500-503, doi:10.1038/377500a0 (1995).

23 Zhang, J., Li, D., Chen, R. \& Xiong, Q. Laser cooling of a semiconductor by 40 kelvin. Nature 493, 504-508, doi:10.1038/nature11721 (2013).

24 Akizuki, N., Aota, S., Mouri, S., Matsuda, K. \& Miyauchi, Y. Efficient near-infrared up-conversion photoluminescence in carbon nanotubes. Nature Communications 6, 8920, doi:10.1038/ncomms9920 (2015).

25 Liu, Q. et al. Single upconversion nanoparticle imaging at sub-10 $\mathrm{W} \mathrm{cm}^{-2}$ irradiance. Nature Photonics 12, 548-553, doi:10.1038/s41566-018-0217-1 (2018).

26 Liang, L. et al. Upconversion amplification through dielectric superlensing modulation. Nature Communications 10, 1391, doi:10.1038/s41467-019-09345-0 (2019).

27 Moody, G., Schaibley, J. \& Xu, X. Exciton dynamics in monolayer transition metal dichalcogenides [Invited]. J. Opt. Soc. Am. B 33, C39-C49, doi:10.1364/JOSAB.33.000C39 (2016).

28 Chernikov, A. et al. Exciton Binding Energy and Nonhydrogenic Rydberg Series in Monolayer WS 2. Physical Review Letters 113, 076802, doi:10.1103/PhysRevLett.113.076802 (2014).

29 Ugeda, M. M. et al. Giant bandgap renormalization and excitonic effects in a monolayer transition metal dichalcogenide semiconductor. Nature Materials 13, 1091-1095, doi:10.1038/nmat4061 (2014).

30 He, K. et al. Tightly Bound Excitons in Monolayer WSe2. Physical Review Letters 113, 026803, doi:10.1103/PhysRevLett.113.026803 (2014).

31 Xia, F., Wang, H., Xiao, D., Dubey, M. \& Ramasubramaniam, A. Two-Dimensional Material 
Nanophotonics. Nat. Photonics 8 (2014).

32 Mak, K. F. \& Shan, J. Photonics and optoelectronics of 2D semiconductor transition metal dichalcogenides. Nat. Photonics 10, 216-226, doi:10.1038/nphoton.2015.282 (2016).

33 Wang, G. et al. Colloquium: Excitons in atomically thin transition metal dichalcogenides. Reviews of Modern Physics 90, 021001, doi:10.1103/RevModPhys.90.021001 (2018).

34 Manzeli, S., Ovchinnikov, D., Pasquier, D., Yazyev, O. V. \& Kis, A. 2D transition metal dichalcogenides. Nat. Rev. Mater. 2, 17033, doi:10.1038/natrevmats.2017.33 (2017).

35 Jones, A. M. et al. Excitonic luminescence upconversion in a two-dimensional semiconductor. Nature Physics 12, 323-327, doi:10.1038/nphys3604 (2016).

36 Jadczak, J. et al. Room temperature multi-phonon upconversion photoluminescence in monolayer semiconductor WS2. Nature Communications 10, 107, doi:10.1038/s41467-018-07994-1 (2019).

37 Törmä, P. \& Barnes, W. L. Strong coupling between surface plasmon polaritons and emitters: a review. Reports on Progress in Physics 78, 013901, doi:10.1088/0034-4885/78/1/013901 (2014).

38 Chikkaraddy, R. et al. Single-molecule strong coupling at room temperature in plasmonic nanocavities. Nature 535, 127-130, doi:10.1038/nature17974 (2016).

39 Tahersima, M. H. et al. Testbeds for Transition Metal Dichalcogenide Photonics: Efficacy of Light Emission Enhancement in Monomer vs Dimer Nanoscale Antennae. ACS Photonics 4, 1713-1721, doi:10.1021/acsphotonics.7b00208 (2017).

40 Sun, J. et al. Light-Emitting Plexciton: Exploiting Plasmon-Exciton Interaction in the Intermediate Coupling Regime. ACS Nano 12, 10393-10402, doi:10.1021/acsnano.8b05880 (2018).

41 Cuadra, J. et al. Observation of Tunable Charged Exciton Polaritons in Hybrid Monolayer $\mathrm{WS}_{2}-$ Plasmonic Nanoantenna System. Nano Letters 18, 1777-1785, doi:10.1021/acs.nanolett.7b04965 (2018).

42 Kleemann, M.-E. et al. Strong-coupling of $\mathrm{WSe}_{2}$ in ultra-compact plasmonic nanocavities at room temperature. Nature Communications 8, 1296, doi:10.1038/s41467-017-01398-3 (2017).

43 Ravindra, N. M. \& Srivastava, V. K. Temperature dependence of the energy gap in semiconductors. Journal of Physics and Chemistry of Solids 40, 791-793, doi:https://doi.org/10.1016/00223697(79)90162-8 (1979).

44 Wang, G. et al. Giant Enhancement of the Optical Second-Harmonic Emission of WSe $\mathrm{W}_{2}$ 
Monolayers by Laser Excitation at Exciton Resonances. Physical Review Letters 114, 097403, doi:10.1103/PhysRevLett.114.097403 (2015).

45 Seyler, K. L. et al. Electrical control of second-harmonic generation in a WSe2 monolayer transistor. Nature Nanotechnology 10, 407-411, doi:10.1038/nnano.2015.73 (2015).

46 Kumar, N. et al. Exciton-exciton annihilation in $\mathrm{MoSe}_{2}$ monolayers. Physical Review B 89, 125427 , doi:10.1103/PhysRevB.89.125427 (2014).

47 Mouri, S. et al. Nonlinear photoluminescence in atomically thin layered WSe 2 arising from diffusion-assisted exciton-exciton annihilation. Physical Review B 90, 155449, doi:10.1103/PhysRevB.90.155449 (2014).

48 Purcell, E. M., Torrey, H. C. \& Pound, R. V. Resonance Absorption by Nuclear Magnetic Moments in a Solid. Physical Review 69, 37-38, doi:10.1103/PhysRev.69.37 (1946).

49 Shan, H. et al. Electron transfer and cascade relaxation dynamics of graphene quantum dots/MoS2 monolayer mixed-dimensional van der Waals heterostructures. Materials Today 24, 10-16 (2019).

50 Qi, P. et al. Remote Lightening and Ultrafast Transition: Intrinsic Modulation of Exciton Spatiotemporal Dynamics in Monolayer MoS2. ACS Nano 14, 6897-6905, doi:10.1021/acsnano.0c01165 (2020).

51 Chen, X. et al. Mode Modification of Plasmonic Gap Resonances Induced by Strong Coupling with Molecular Excitons. Nano Letters 17, 3246-3251, doi:10.1021/acs.nanolett.7b00858 (2017).

52 Palik, E. D. Handbook of optical constants of solids. Vol. 3 (Academic press, 1998).

53 Johnson, P. B. \& Christy, R.-W. Optical constants of the noble metals. Physical review B 6, 4370 (1972).

54 Jung, G.-H., Yoo, S. \& Park, Q. H. Measuring the optical permittivity of two-dimensional materials without a priori knowledge of electronic transitions. Nanophotonics 8, 263-270, doi:doi:10.1515/nanoph-2018-0120 (2019).

\section{Acknowledgements}

This work is supported by the National Key Research and Development Program of China (grant nos. 2020YFA0211300, 2017YFA0205700, 2017YFA0206000, and 2019YFA0210203), National Science Foundation of China (grant nos. 12027807, 11674012, 61521004, 21790364, 61422501, and 
486 11374023), Beijing Natural Science Foundation (grant nos. Z180011 and L140007), Foundation for 487 the Author of National Excellent Doctoral Dissertation of PR China (grant no. 201420), National 488 Program for Support of Top-notch Young Professionals (grant no. W02070003), High-performance 489 Computing Platform of Peking University, and Project funded by China Postdoctoral Science 490 Foundation (2019M660283).

491 Author contributions

492 Z.F. supervised the project. P.Q., Y.L. and Y.D. performed all experiments. P.Q. accomplished all 493 simulations and wrote the paper. G.T. fabricated the monolayer WSe2. All authors contribute to the 494 scientific discussions and paper revisions.

495 Additional information

496 Supplementary information is available in the online version of the paper. Reprints and permissions 497 information is available online at www.nature.com/reprints. Publisher's note: Springer Nature remains 498 neutral with regard to jurisdictional claims in published maps and institutional affiliations. 499 Correspondence and requests for materials should be addressed to Z.F.

500 Competing financial interests

501 The authors declare no competing financial interests. 


\section{Supporting Information}

\section{Giant excitonic upconverted emission of two-dimensional semiconductor in doubly resonant plasmonic nanocavity}

Pengfei Qi, ${ }^{1, \#}$ Yuchen Dai, ${ }^{1, \#}$ Yang Luo, ${ }^{1, \#}$ Guangyi Tao, ${ }^{1,2}$ Liheng Zheng, ${ }^{1}$ Tianhao Zhang, ${ }^{2}$ Bo Shen, ${ }^{1}$ Feng Lin, ${ }^{1}$ Zheyu Fang ${ }^{1, *}$

${ }^{1}$ School of Physics, State Key Laboratory for Mesoscopic Physics, Academy for Advanced Interdisciplinary Studies, Collaborative Innovation Center of Quantum Matter, Nano-optoelectronics Frontier Center of Ministry of Education, Peking University, Beijing 100871, China

${ }^{2}$ Photonics Research Center, School of Physics, MOE Key Lab of Weak-Light Nonlinear Photonics, and Tianjin Key Lab of Photonics Materials and Technology for Information Science, Nankai University, Tianjin 300071, China

\# These authors contributed equally: Pengfei Qi, Yuchen Dai and Yang Luo

*Email: zhyfang@pku.edu.cn 


\section{CONTENTS}

S1. SEM images of Au nanocubes and plasmonic cavities ..................................... 1

$\mathrm{S} 2$. Raman spectra of monolayer $\mathrm{WSe}_{2}$ in free space and plasmonic cavity ................2

S3. Temperature-dependent upconverted emission spectra .......................................

S4. Energy density-dependent PL spectra of monolayer WSe $\mathrm{WS}_{2} \ldots \ldots \ldots \ldots \ldots \ldots \ldots \ldots \ldots \ldots \ldots . . . . . \ldots$

S5. Estimation of real saturated excitation energy density ......................................

S6. Schematics for simulating far-field radiation pattern ........................................6

S7. Far-field radiation for monolayer $\mathrm{WSe}_{2}$ in free space and plasmonic cavity ..........7

S8. Charge and field distribution around plasmonic cavity at $1.98 \mathrm{eV}$...................... 8

S9. Magnetic field distributions around plasmonic cavity.......................................

S10. Exciton relaxation for the monolayer $\mathrm{WSe}_{2}$ on $\mathrm{Au}$ film................................... 10

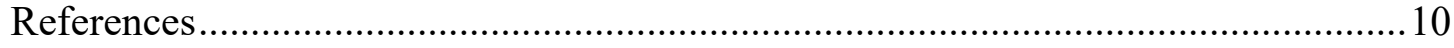




\section{S1. SEM images of Au nanocubes and plasmonic cavities}

a

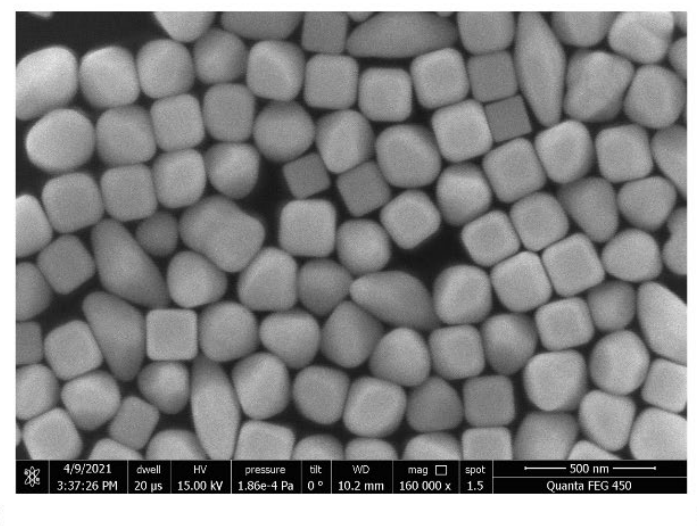

b

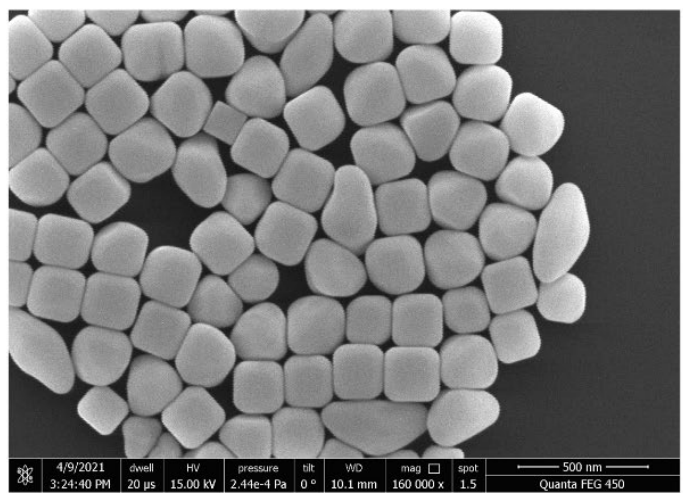

$c$

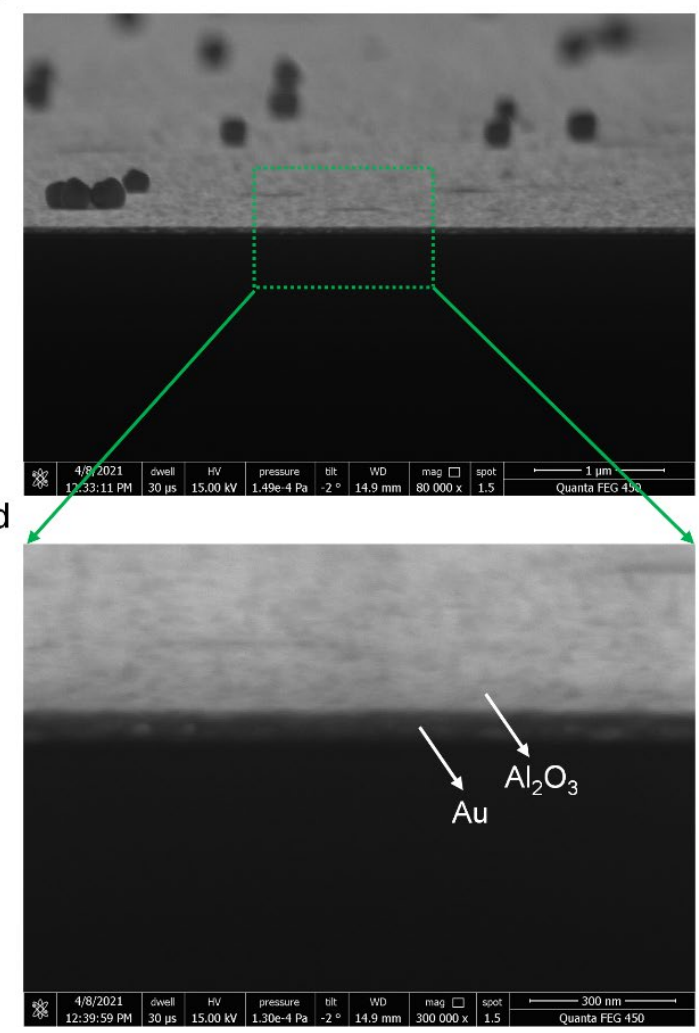

Figure S1. SEM images of Au nanocubes and plasmonic cavities. (a), (b) Top-view SEM images of |the seed-mediated synthetic Au nanocubes. (c), (d) Cross-sectional $\mathrm{SEM}$ view of a typical Au nanocube $/ \mathrm{Al}_{2} \mathrm{O}_{3} / \mathrm{Au}$ film plasmonic cavities heterostructure, imaged at a $2^{\circ}$ tilted angle. The dark area corresponds to the substrate of $\mathrm{Si} / \mathrm{SiO}_{2}$. 


\section{S2. Raman spectra of monolayer $\mathrm{WSe}_{2}$ in free space and plasmonic cavity}

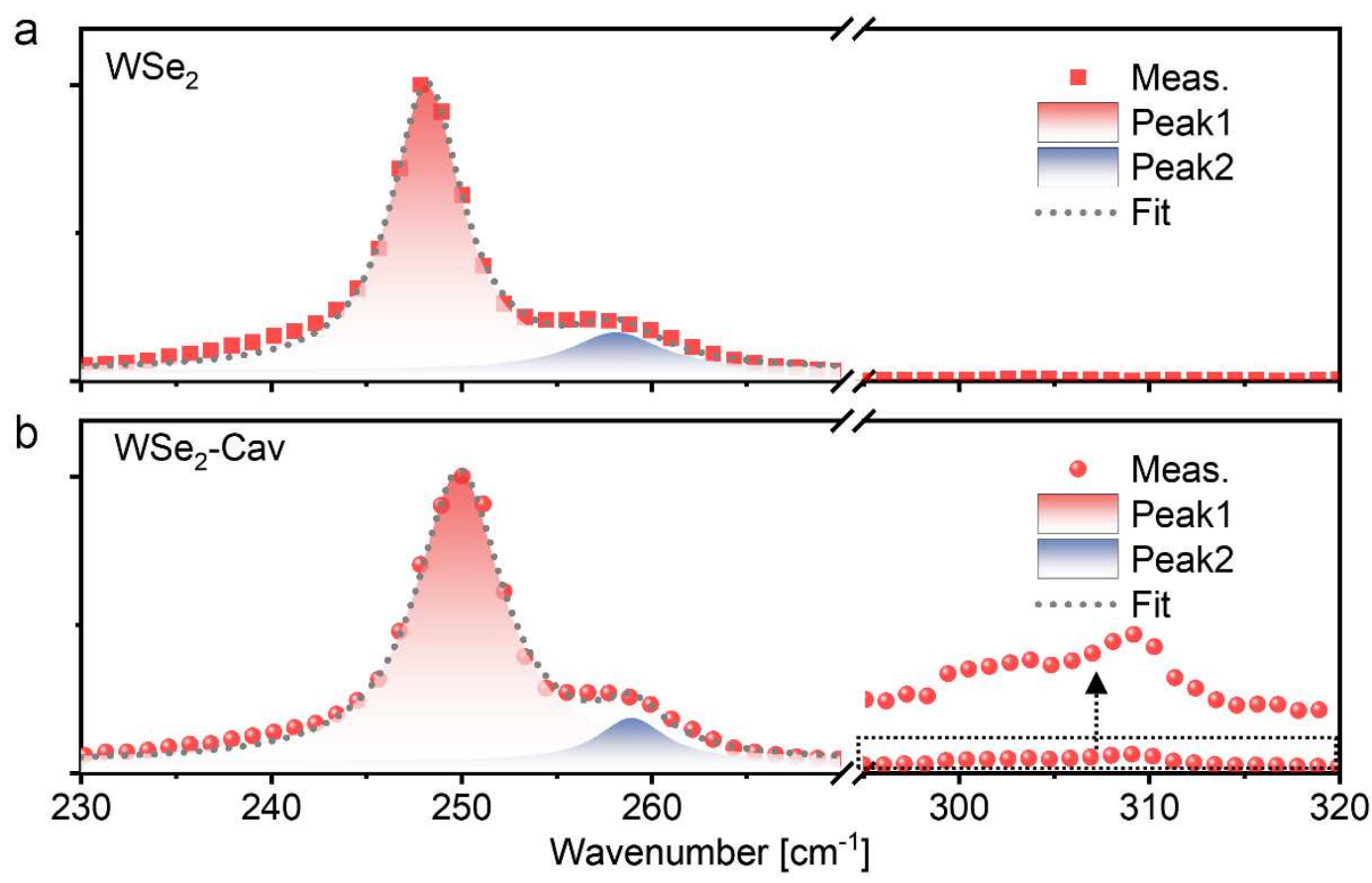

Figure S2. Raman spectra and corresponding Lorenz fitting of the monolayer $\mathrm{WSe}_{2}$ in free space (a) and plasmonic cavity (b). The monolayer $\mathrm{WSe}_{2}$ in free space has a typical Raman spectrum with two dominant peaks at $248.19 \mathrm{~cm}^{-1}$ (phonon energy of 30.78 $\mathrm{meV}$ ) and $258.16 \mathrm{~cm}^{-1}$ (phonon energy of $32.01 \mathrm{meV}$ ) corresponding to atomic displacements of the in-plane ${ }^{E_{2 g}^{1}}$ and out-of-plane ${ }^{A_{1 g}}$ modes (a), respectively. There are slightly blueshifts of $1.71 \mathrm{~cm}^{-1}$ and $0.82 \mathrm{~cm}^{-1}$ for the monolayer WSe2 plasmonic cavity (b). The peaks located at $303 \mathrm{~cm}^{-1}$ and $309 \mathrm{~cm}^{-1}$ in (b) can be attributed to the poly(sodium-p-styrenesulfonate) (PSS). 


\section{S3. Temperature-dependent upconverted emission spectra}
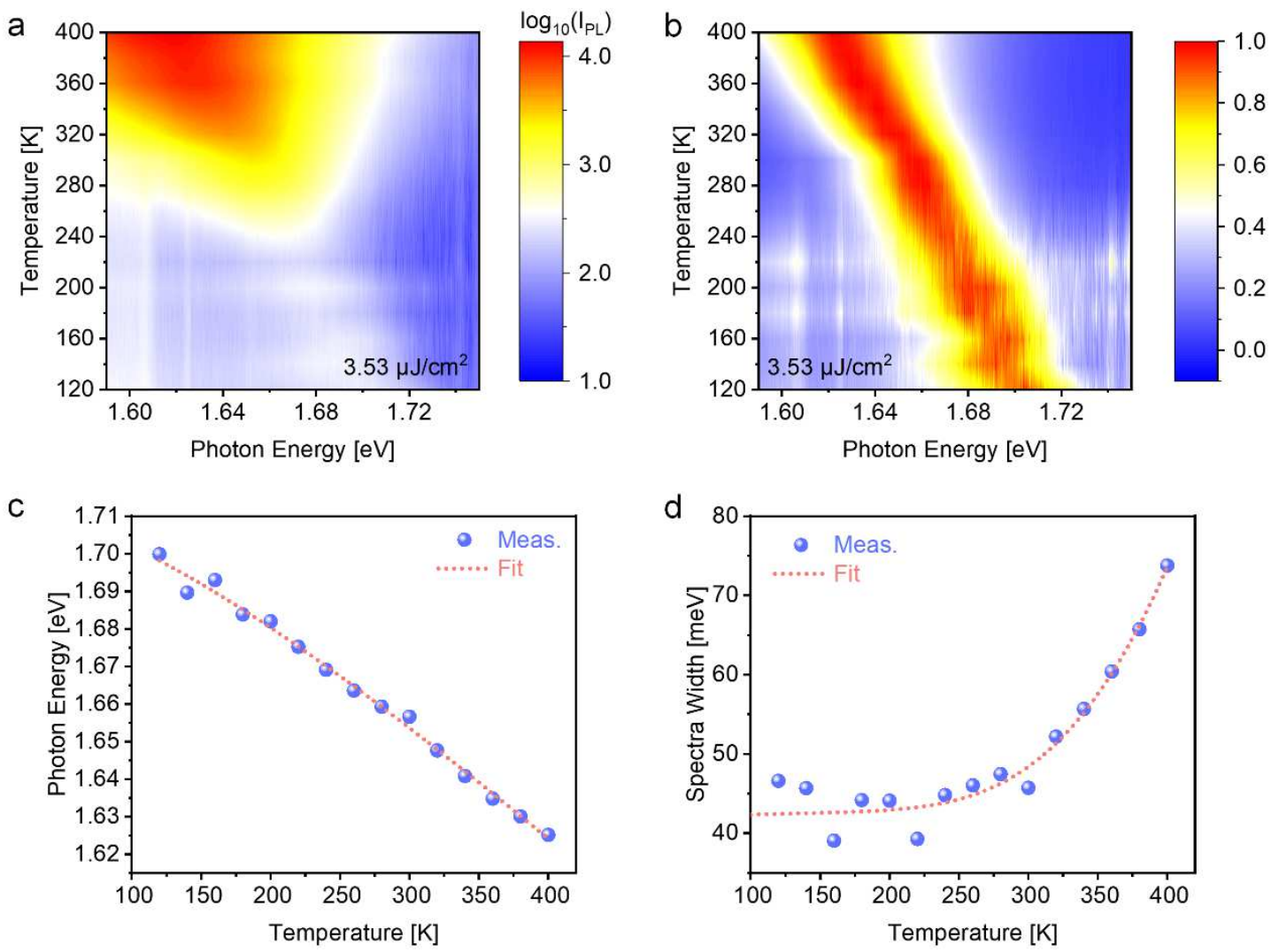

Figure S3. Temperature-dependent upconverted emission spectra. (a) Temperature dependent PL spectra for excitation photon energy at $1.52 \mathrm{eV}$. (b) Normalized PL spectra of (a). (c), (d) Evolution of peak energy and spectral width with temperature.

The variation of exciton peak can be attributed to temperature-dependent lattice dilatation and electron-phonon interaction. The exciton peak shift (Figure S3c) can be well fitted by the well-known Varshni equation, $E_{g}(T)=E_{g}(0)-\alpha T^{2} /(T+\beta)$, which describes the temperature-dependence of energy gap for various semiconductors. ${ }^{1}$ The global optimal parameters of fitting curves are $E_{g}(0)=1.712 \mathrm{eV}, \alpha=3.57 \times 10^{-4} \mathrm{eV} / \mathrm{K}$ and $\beta=249.3 \mathrm{~K}$. The broadening excitons linewidth results from the interaction of excitons with the longitudinal-acoustical (LA) and longitudinal-optical (LO) phonon modes of lattice for semiconductor without considerable impurity doping and defects. Accordingly, the temperature-dependent linewidth of excitons can be written as $\Gamma(T)=$ 
$\Gamma_{0}+\gamma_{\mathrm{LA}} T+\gamma_{\mathrm{LO}} N_{\mathrm{LO}}(T),{ }^{2}$ where the second term describes the contribution of the interaction between excitons and LA phonons, the third term describes derive from the interaction between excitons and LO phonons with $N_{\mathrm{LO}}(T)$ represents the $\mathrm{LO}$ phonons occupation with Bose-Einstein distribution, and the constant term arises from scattering due to intrinsic imperfections. As depicted by the red dotted line in Figure S3d, temperature-dependent linewidth can be strictly described by $\Gamma(T)$, where $\Gamma_{0}=41.92$ $\mathrm{meV}, \gamma_{\mathrm{LA}}=0.04 \mathrm{meV} / \mathrm{K}$, and $\gamma_{\mathrm{LO}}=5.64 \mathrm{meV}$.

\section{S4. Energy density-dependent PL spectra of monolayer WSe 2}

a

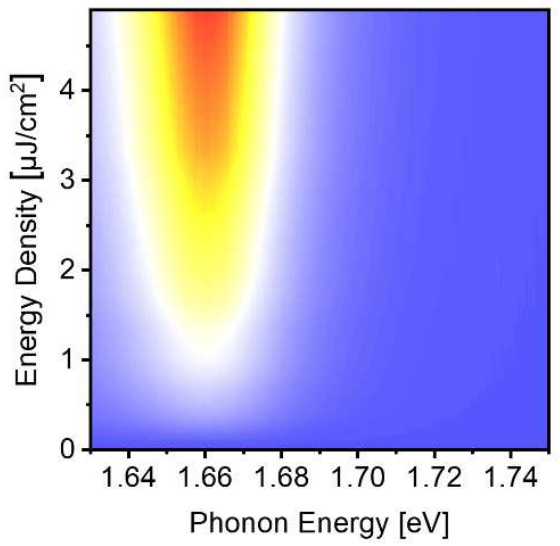

b

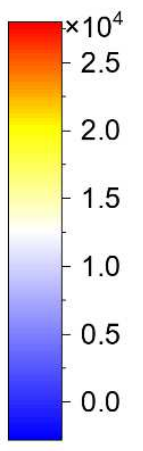

Figure S4. Energy density-dependent PL spectra of monolayer $\mathrm{WSe}_{2}$ for excitation photon energy of $3.04 \mathrm{eV}$. (a) Excitation energy density-dependent unconverted PL spectra for monolayer WSe2. (b) Exitation energy density-dependent integrated unconverted PL intensity for monolayer WSe2, which can be well fitted by equation (S1). The saturated upconverted emission intensity $I_{\text {sat }}=1.4 \times 10^{7}$ counts for monolayer $\mathrm{WSe}_{2}$ can be obtained. 


\section{S5. Estimation of real saturated excitation energy density}

a

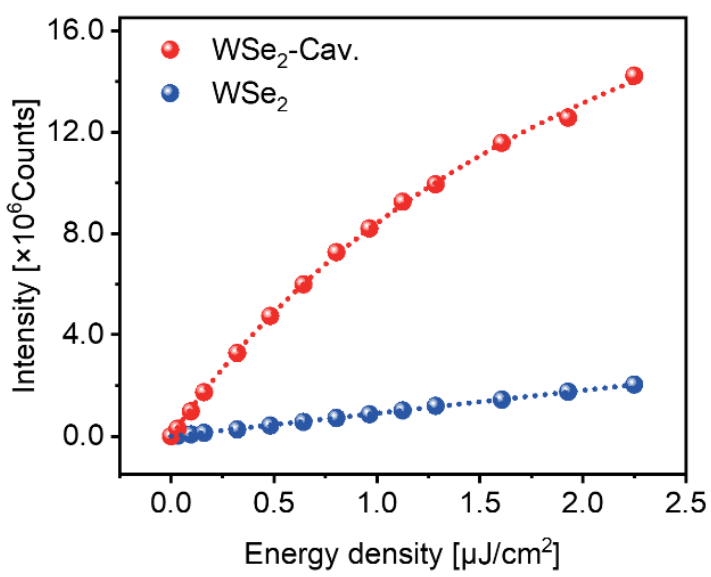

b

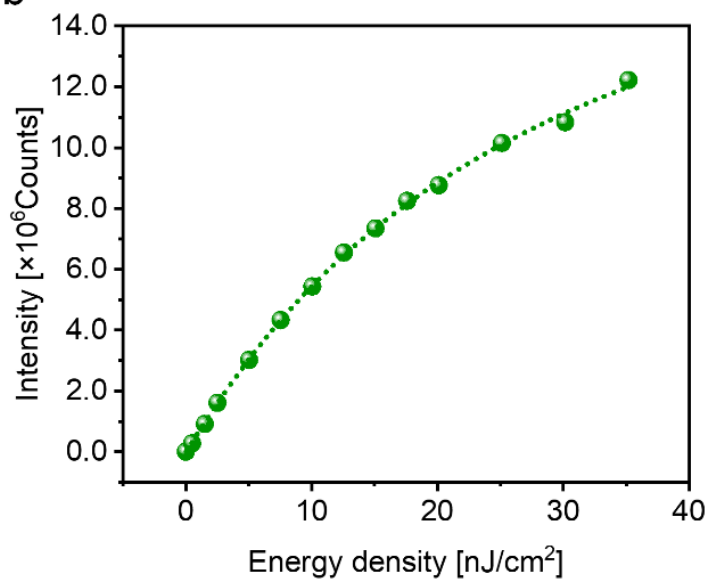

Figure S5. Estimation of real saturated excitation energy density. (a) Exitation energy density-dependent integrated unconverted PL intensity for monolayer $\mathrm{WSe}_{2}$ in free space and plasmonic cavity. (b) Actual exitation energy density-dependent integrated unconverted PL intensity for monolayer $\mathrm{WSe}_{2}$ in designed plasmonic cavity.

The dependence of upconversion intensity on excitation energy density for monolayer $\mathrm{WSe}_{2}$ in free space and plasmonic cavity in Figure S5a can be well fitted by

$$
I=I_{\text {sat }} \frac{f}{f+f_{\text {sat }}}
$$

where $I$ and $I_{\text {sat }}$ are total upconverted emission intensity and the corresponding saturation value, $f$ and $f_{\text {sat }}$ are the excitation energy density and the corresponding saturation value, respectively.

In light of the fact that the collected PL spectra in plasmonic cavity (Figure S5a) are comprised of the excitons coupled to plasmonic cavity and neighbouring excitons without coupling, the measured dependence of upconversion intensity on excitation energy density for plasmonic upconverter devices can be revised as following

$$
I_{\text {meas }}=I_{c a v}+I_{\text {free }}=I_{\text {sat-cav }} \frac{p f}{p f+f_{\text {sat-cav }}}+I_{\text {sat-free }} \frac{(1-p) f}{(1-p) f+f_{\text {sat-cav }}}
$$


Where $I_{c a v}$ and $I_{\text {free }}$ are the PL intensities of the excitons coupled to plasmonic cavity and neighbouring excitons without coupling, $I_{\text {sat-cav }}$ and $I_{\text {sat-free }}$ are the saturated PL intensities for plasmonic cavity and neighbouring excitons without coupling, $f_{\text {sat-cav }}$ and $f_{\text {sat-cav }}$ are the saturated excitation energy density for plasmonic cavity and neighbouring excitons without coupling, $p$ is the proportion of the actual excitation photon energy used to excite the exciton in plasmonic cavity, which can be approximate as $S_{P C} / S_{0}, S_{0}$ defines the excitation area in our measurements, while $S_{P C}$ represents the hotspot area in plasmonic cavity.

Finally, the actual exitation energy density-dependent integrated unconverted PL intensity for monolayer $\mathrm{WSe}_{2}$ in plasmonic cavity can be calculated from Figure S5(a), as shown in Figure S5(b). Accordingly, the real saturated excitation energy density in our designed doubly resonant plasmonic cavity can be estimated as $32.9 \mu \mathrm{J} / \mathrm{cm}^{2}$, which is reduced by $2 \sim 3$ orders of magnitude compared with free space.

\section{S6. Schematics for simulating far-field radiation pattern}

a

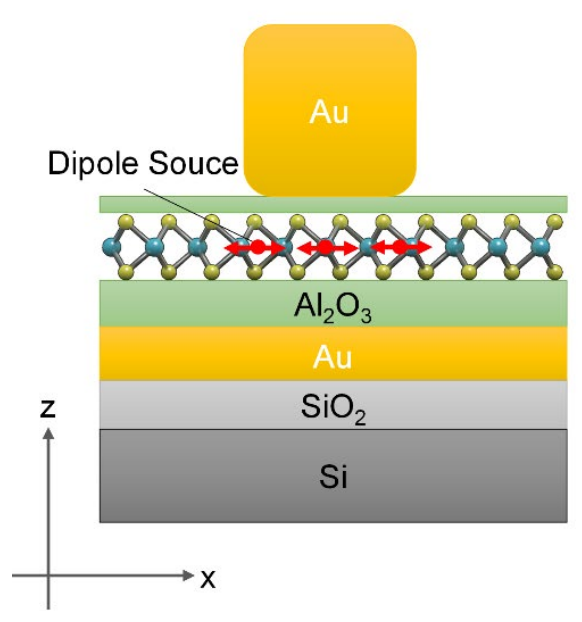

b

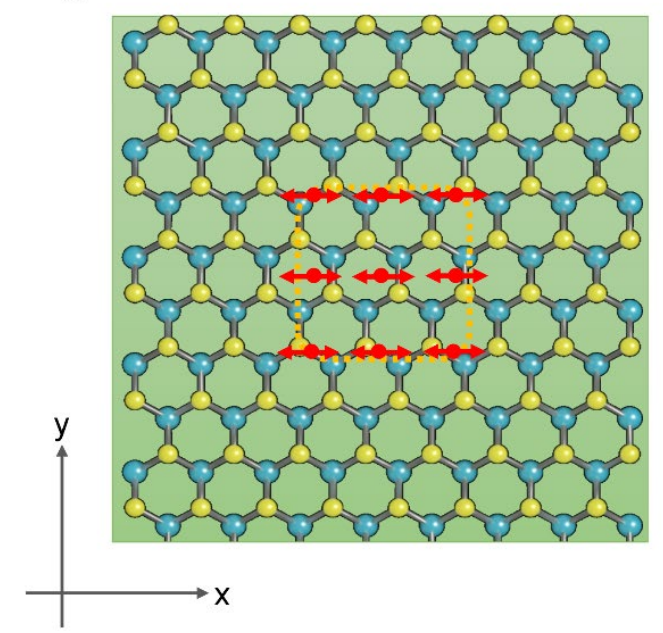

Figure S6. Schematics for simulating far-field radiation pattern. The in-plane dipole source arrays with center wavelength of $750 \mathrm{~nm}$ and spectral width of $25 \mathrm{~nm}$ were adopted. 


\section{S7. Far-field radiation for monolayer $\mathrm{WSe}_{2}$ in free space and plasmonic cavity}

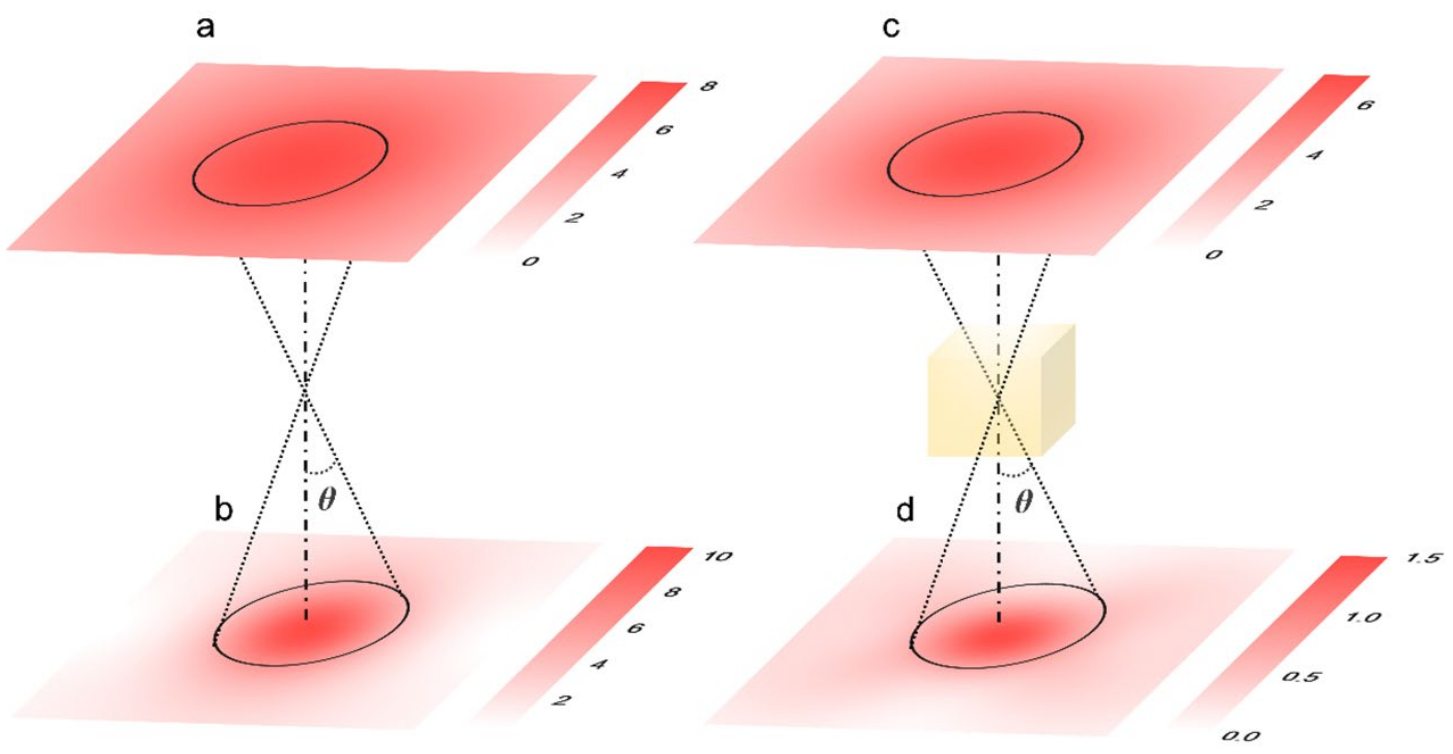

Figure S7. Far-field radiation patterns $\left(\left|\mathrm{E} / \mathrm{E}_{0}\right|\right)$ for monolayer $\mathrm{WSe}_{2}$ in free space and plasmonic cavity. (a), (b) Far-field radiation patterns for a monitor placed on the top (a) and bottom (b) of the monolayer WSe2. (c), (d) Far-field radiation patterns for a monitor placed on the top (c) and bottom (d) of the monolayer $\mathrm{WSe}_{2}$ in plasmonic cavity. The average intensity around the angle $\theta$ can be calculated and plotted as the far-field angular radiation patterns in Figures $4 \mathrm{~b}$ and $4 \mathrm{c}$. 
S8. Charge and field distribution around plasmonic cavity at $1.98 \mathrm{eV}$

a

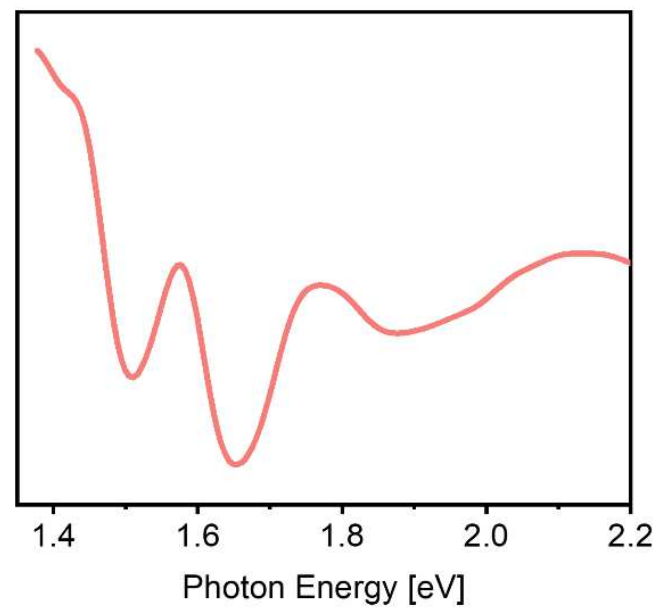

C
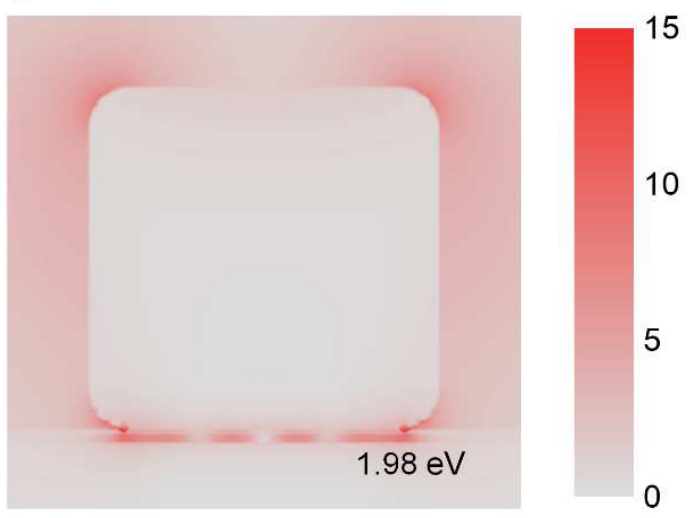

b

$1.98 \mathrm{eV}$

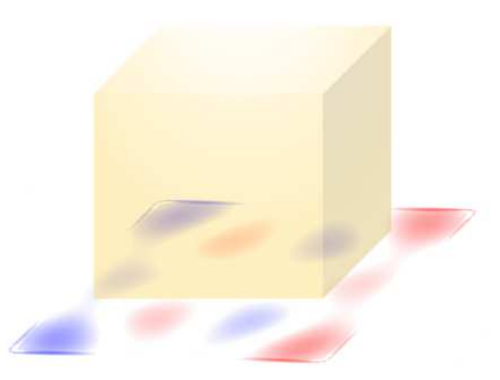

d

Figure S8. (a) Reflectance spectra of plasmonic upconverter devices simulated by the finite-difference time-domain (FDTD) method. (b), (c), (d) charge and field distribution $\left(\left|\mathrm{E} / \mathrm{E}_{0}\right|\right)$ around plasmonic cavity for the mode at $1.98 \mathrm{eV}$. 
S9. Magnetic field distributions around plasmonic cavity

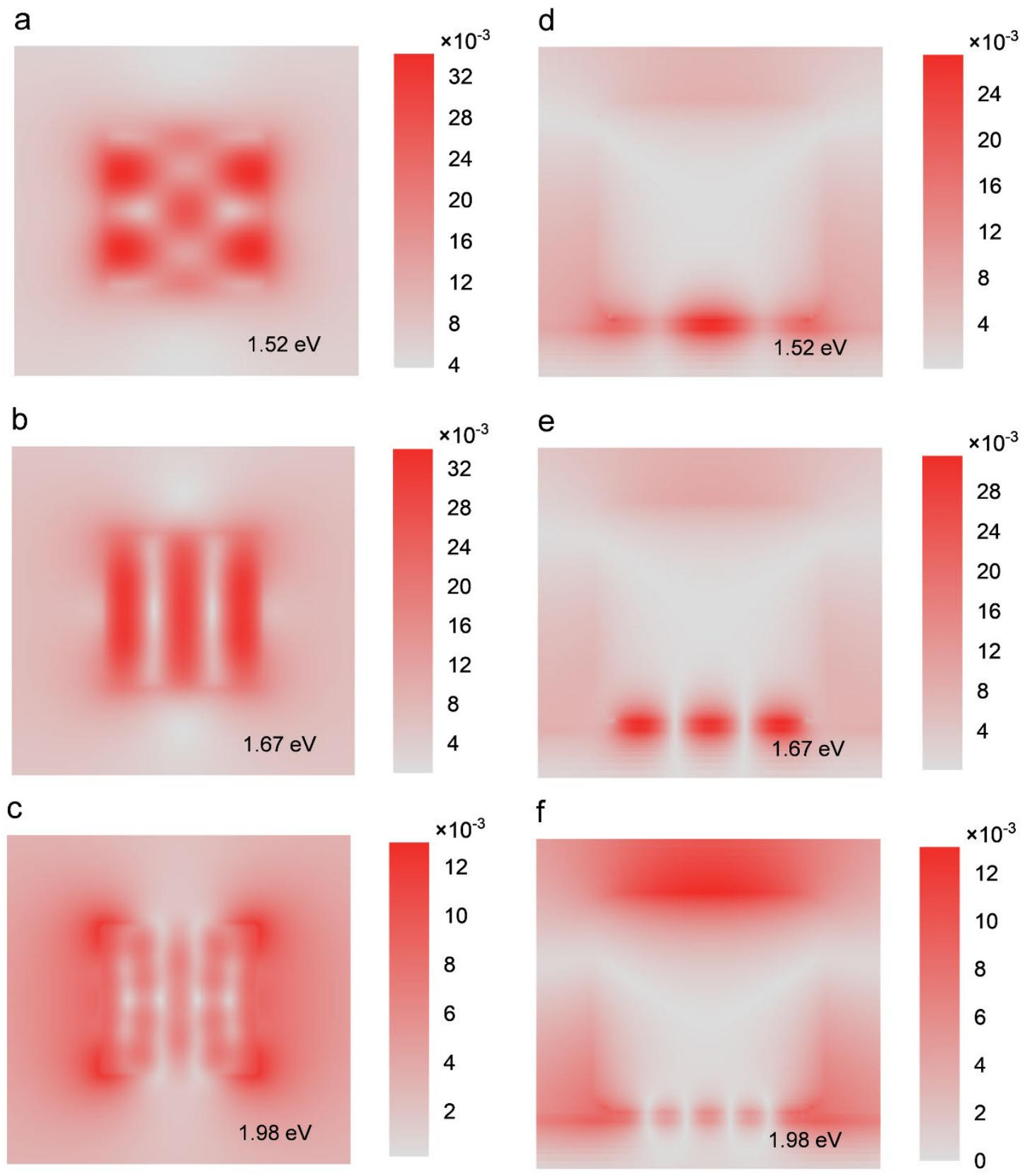

Figure S9. Magnetic field distributions around plasmonic cavity. (a), (d) Magnetic field distributions around plasmonic cavity at $1.52 \mathrm{eV}$. (b), (e) Magnetic field distributions around plasmonic cavity at $1.67 \mathrm{eV}$. (c), (f) Magnetic field distributions around plasmonic cavity at $1.98 \mathrm{eV}$. (a), (b), (c) corresponds to the magnetic field distributions at the monolayer $\mathrm{WSe}_{2}$ plane, and (d), (e), (f) corresponds to the magnetic field distributions at the xz plane 


\section{S10. Exciton relaxation for the monolayer $\mathrm{WSe}_{2}$ on $\mathrm{Au}$ film}
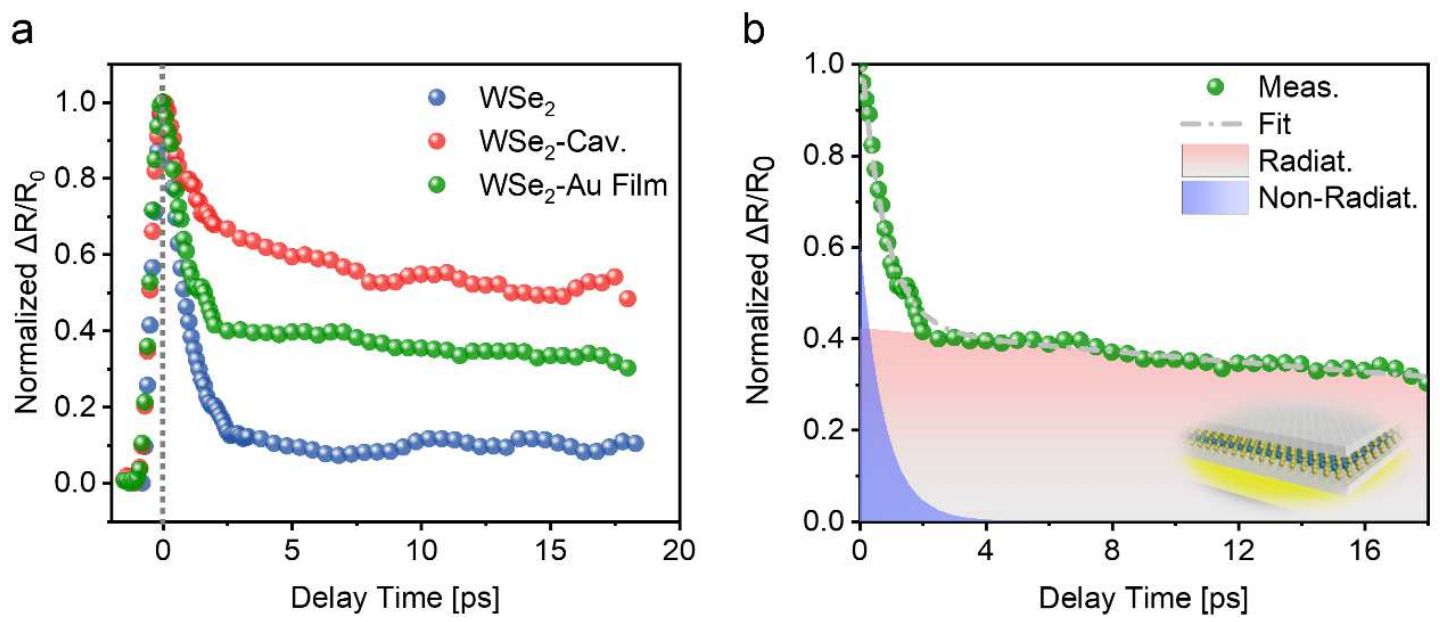

Figure S10. (a) Normalized differential reflection signal $\Delta R / R_{0}$ for the monolayer $\mathrm{WSe}_{2}$ on $\mathrm{Au}$ film (green points). (b) Fittings of the exciton relaxation for the monolayer $\mathrm{WSe}_{2}$ on $\mathrm{Au}$ film. The monolayer $\mathrm{WSe}_{2}$ on $\mathrm{Au}$ film undergoes the exciton relaxation with a fast decay time constant of $0.76 \mathrm{ps}$ and a slow decay time constant of $62 \mathrm{ps}$.

\section{References}

1 Varshni, Y. P. Temperature dependence of the energy gap in semiconductors. Physica 34, 149-154, doi:https://doi.org/10.1016/0031-8914(67)90062-6 (1967).

2 Rudin, S., Reinecke, T. L. \& Segall, B. Temperature-dependent exciton linewidths in semiconductors. Physical Review B 42, 11218-11231, doi:10.1103/PhysRevB.42. $11218(1990)$. 


\section{Figures}

a

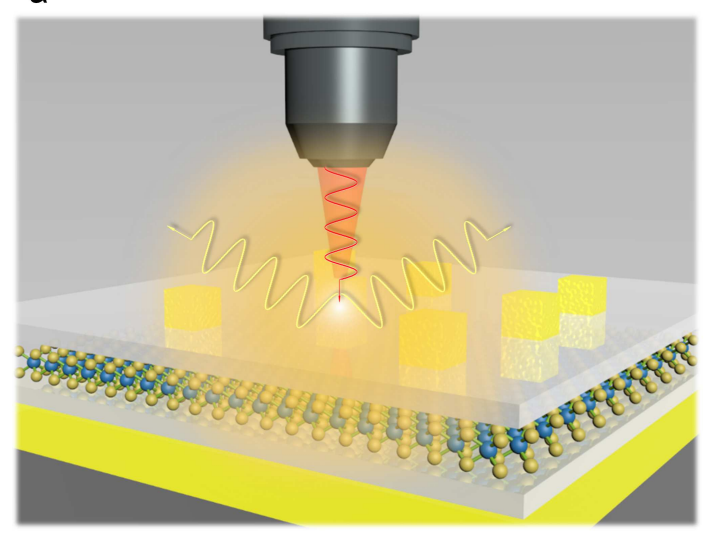

b

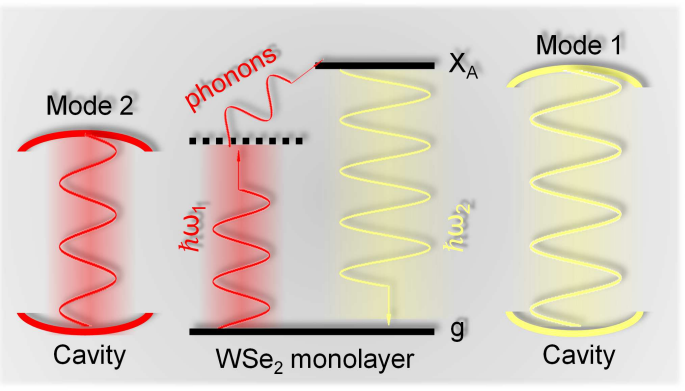

C

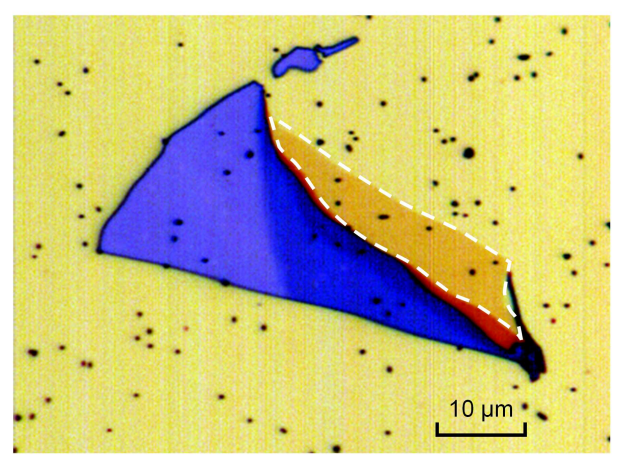

d

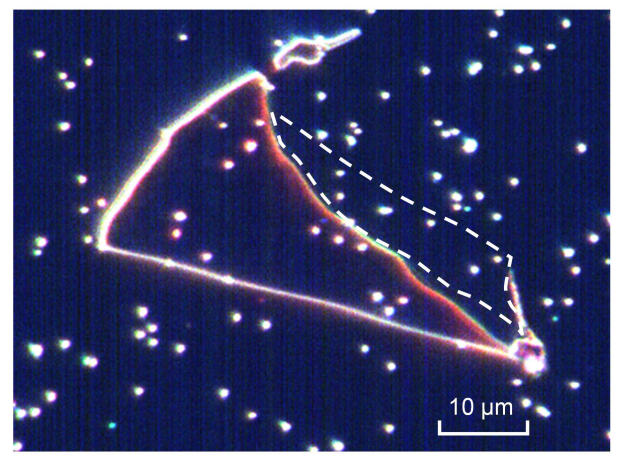

e
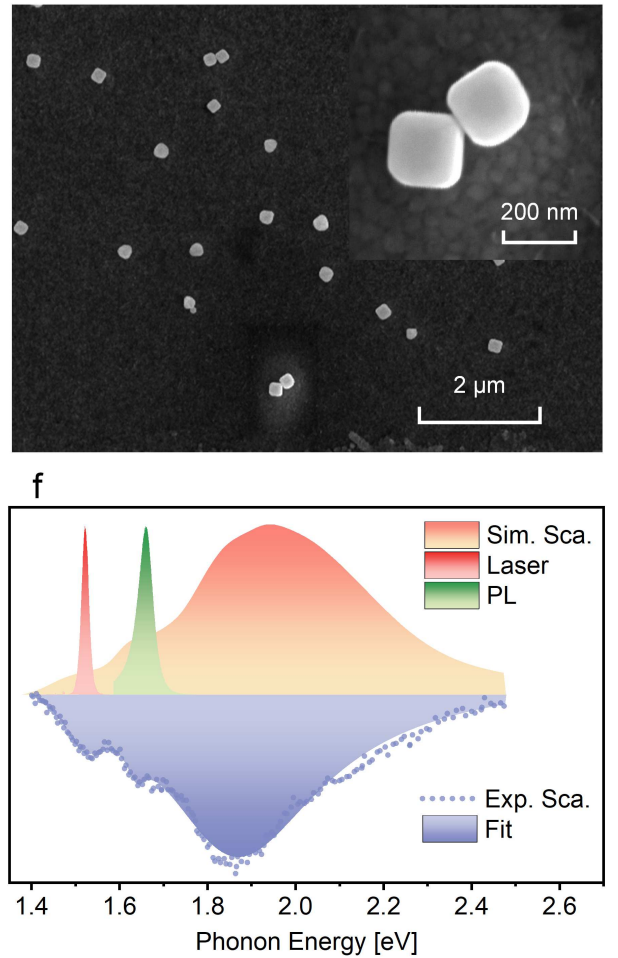

Figure 1

Design and characterization of plasmonic upconverter devices. (a) Schematic of the designed Au nanocube/WSe2/substrate plasmonic upconverter devices. (b) Desired band alignment diagram of monolayer WSe2 in plasmonic nanocavities and the photon upconversion process of 2D excitons. (c) Bright- and (d) dark-field microscope optical images of a representative sample. (e) Scanning electron micrograph of $170 \mathrm{~nm}$ Au nanocube on substrate, and the inset shows the zoom-in image from the top view. (f) Excitation laser and monolayer WSe2 PL spectra overlap with simulated scattering spectrum of a plasmonic nanocavity, which is consistent with experimental results. The excitation laser and upconverted emission spectra doubly resonant with plasmonic cavity modes at $\lambda \approx 1.52 \mathrm{eV}$ and $1.67 \mathrm{eV}$, respectively. 
a

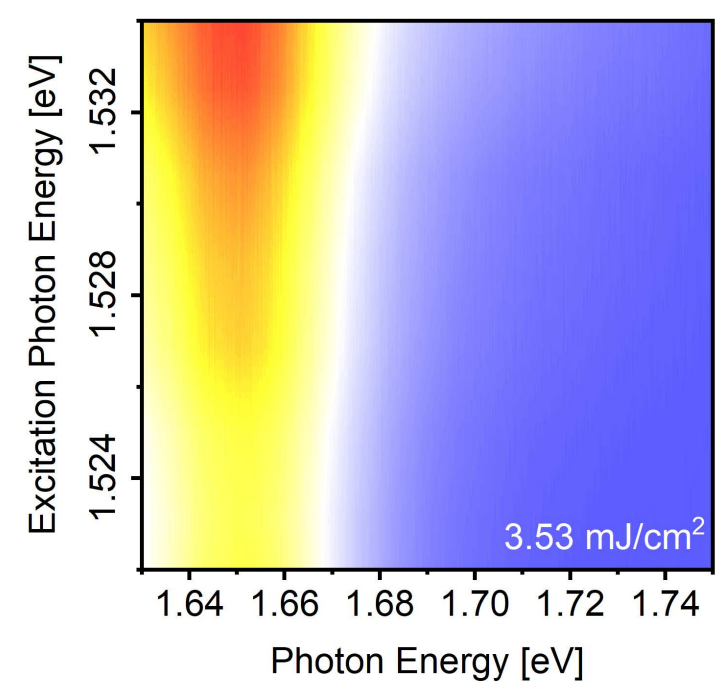

b

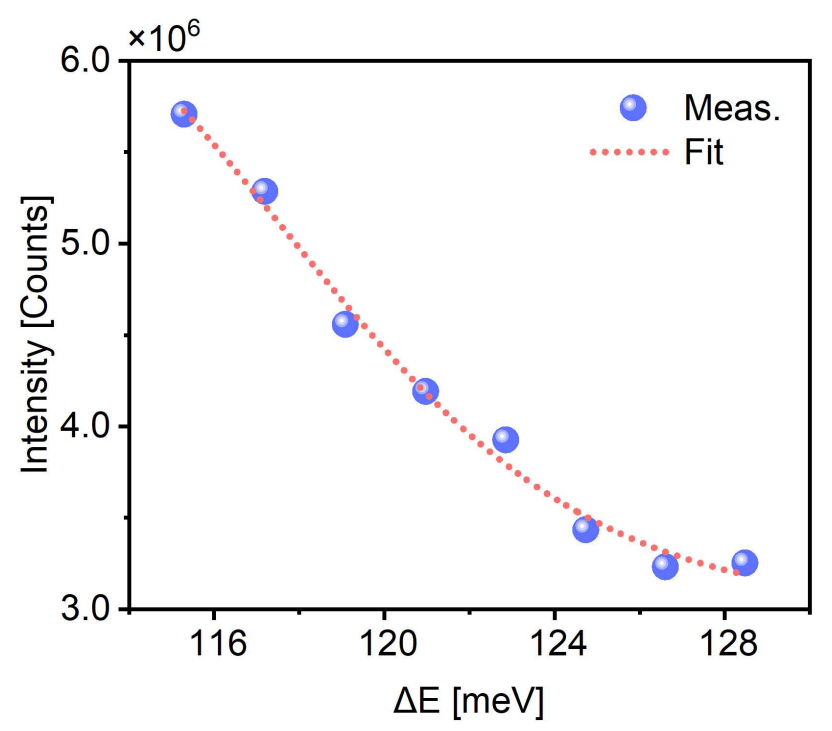

C

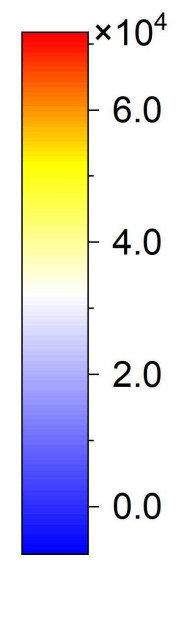

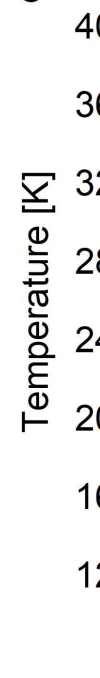

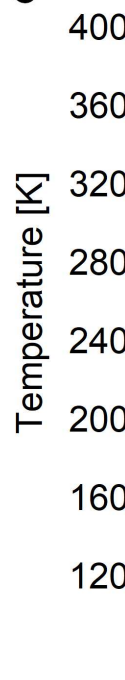

d

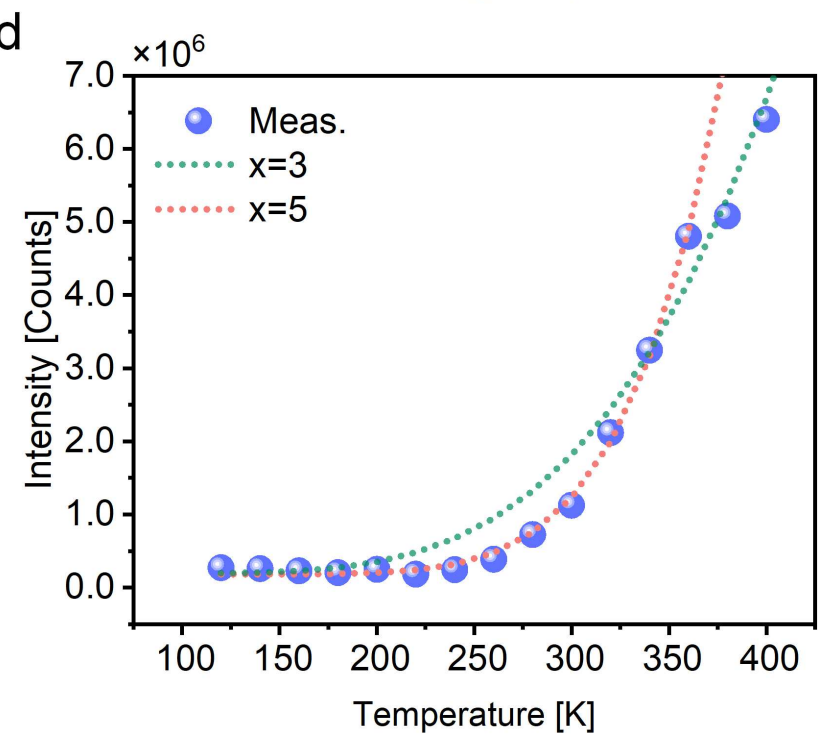

Figure 2

Phonon-assisted excitonic upconverted emission of monolayer WSe2. (a) Excitation photon energydependent PL spectra. (b) Excitation photon energy-dependent integrated PL intensity calculated from (a). (c) Temperature-dependent PL spectra for excitation photon energy at $1.52 \mathrm{eV}$. (d) Temperaturedependent integrated PL intensity calculated from (c). 
a

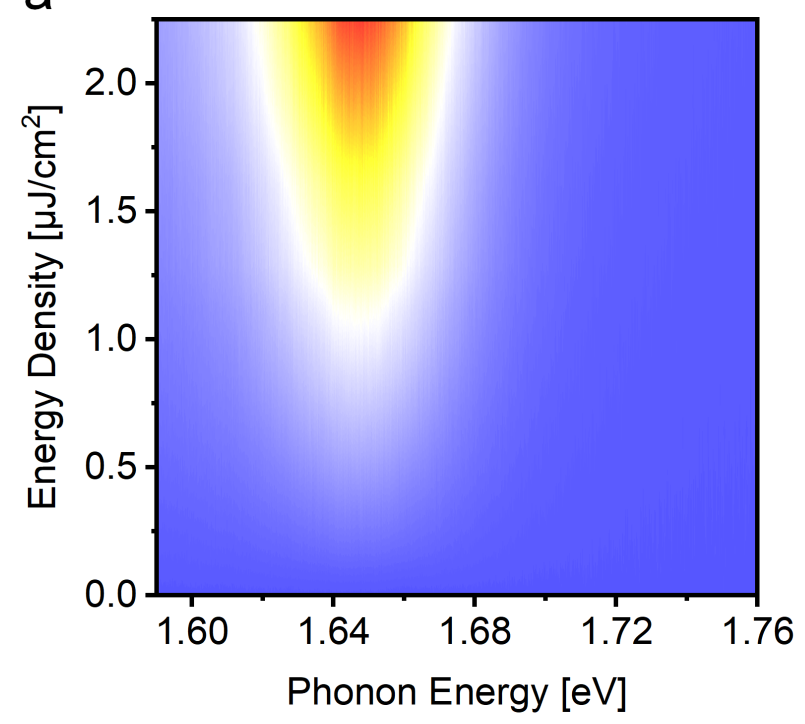

C

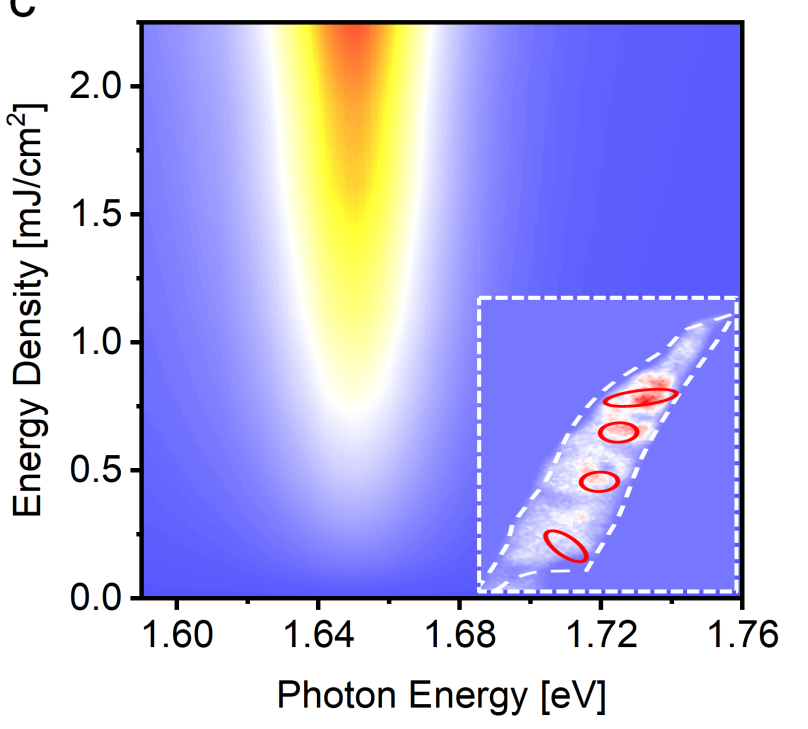

b
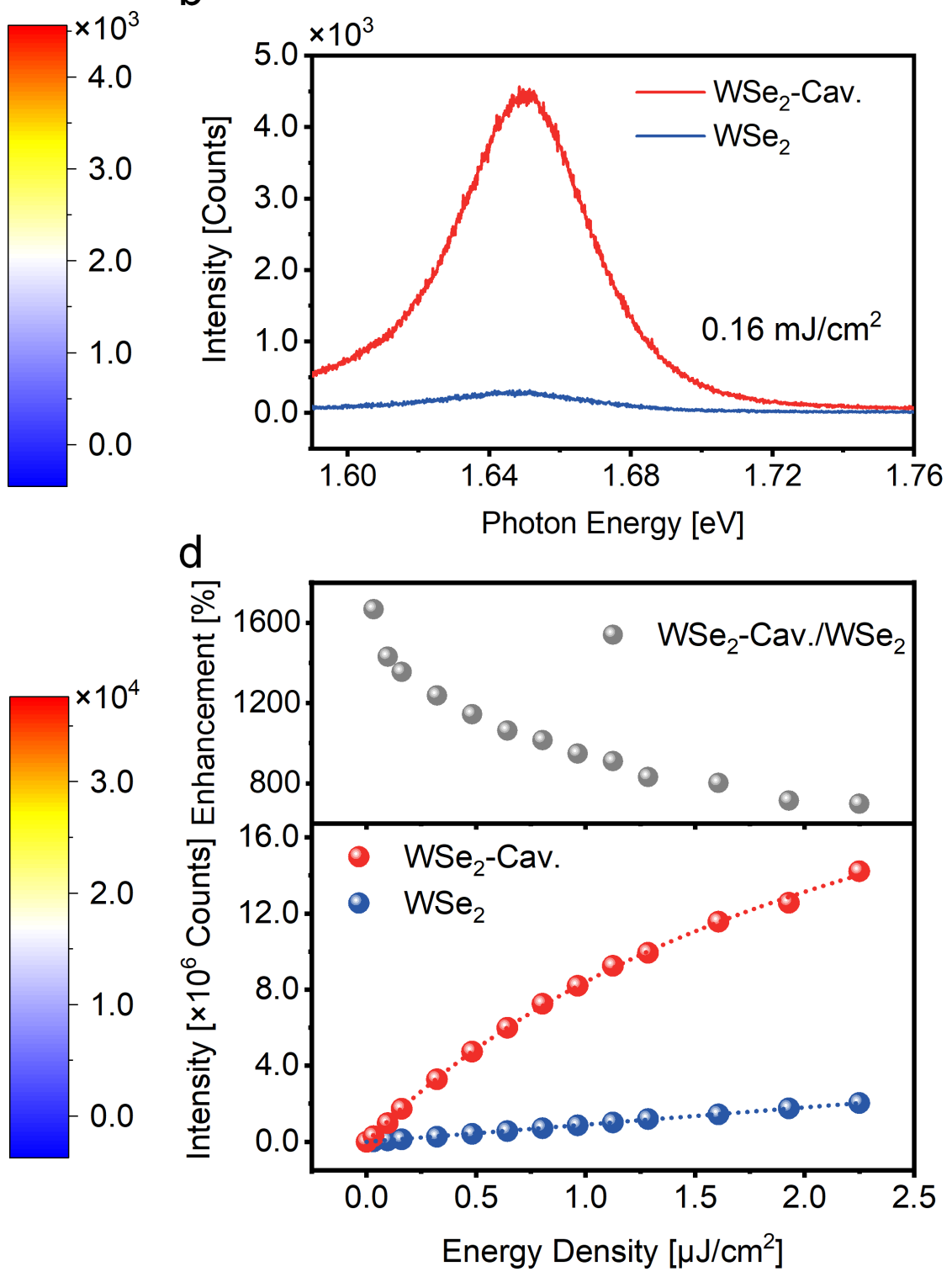

Figure 3

Upconversion amplificated by plasmonic cavity. (a) Excitation energy density-dependent unconverted PL spectra for monolayer WSe2 on SiO2/Si. (b) Excitation energy density-dependent unconverted PL spectra for monolayer WSe2 in designed plasmonic cavity. (c) Enhanced unconverted PL spectra of monolayer WSe2 in designed plasmonic cavity at the excitation energy density of $0.16 \mathrm{~mJ} / \mathrm{cm} 2$. Inset: PL maps of the plasmonic nanocavity effect on exciton emission, where the nanocubes and the monolayer WSe2 are marked by red solid line and white dashed line. (d) Exitation energy density-dependent integrated unconverted PL intensity (top) and the enhancement (down) for monolayer WSe2 in designed plasmonic cavity. 
a

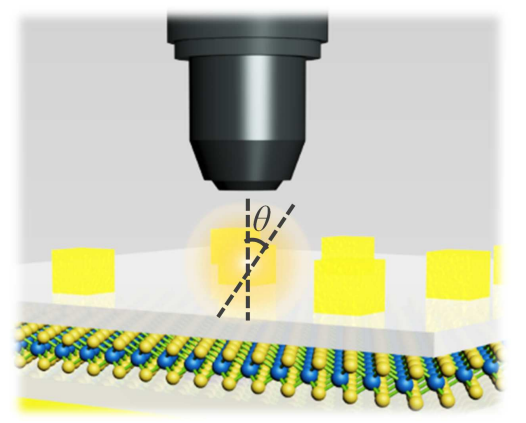

d

$1.67 \mathrm{eV}$

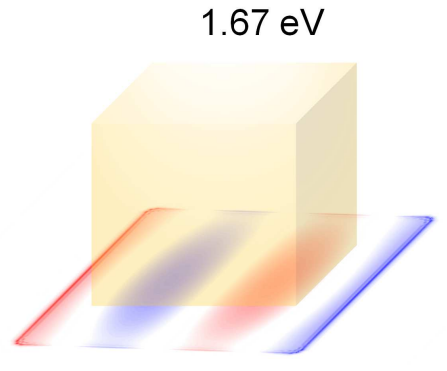

b

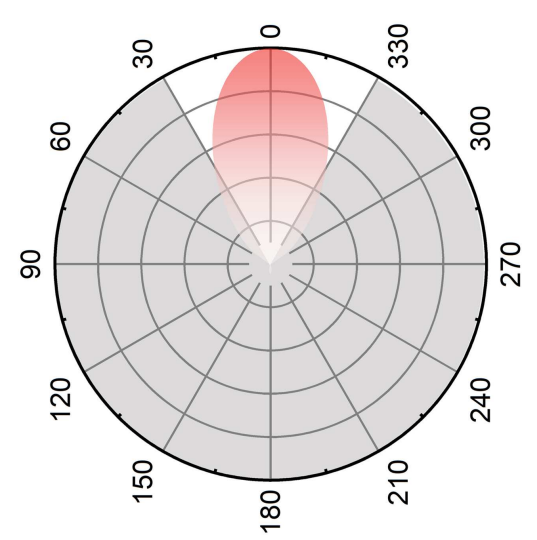

C

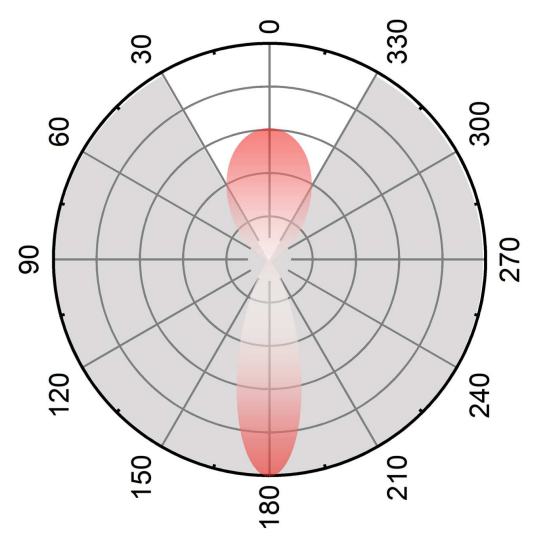

g $1.52 \mathrm{eV}$

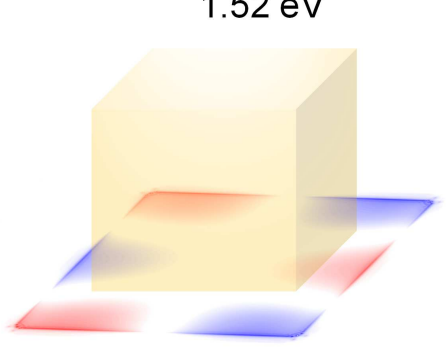

e

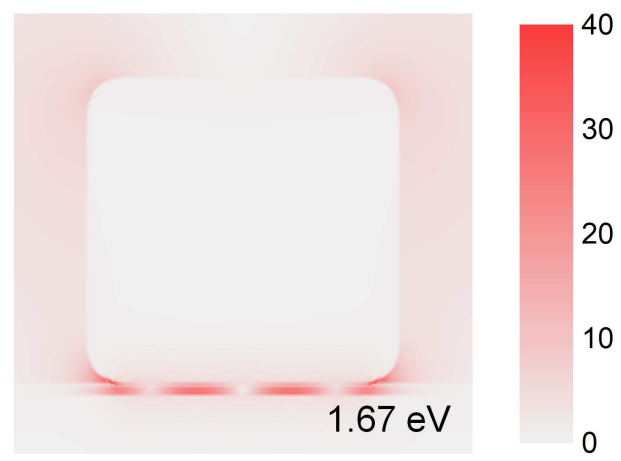

h

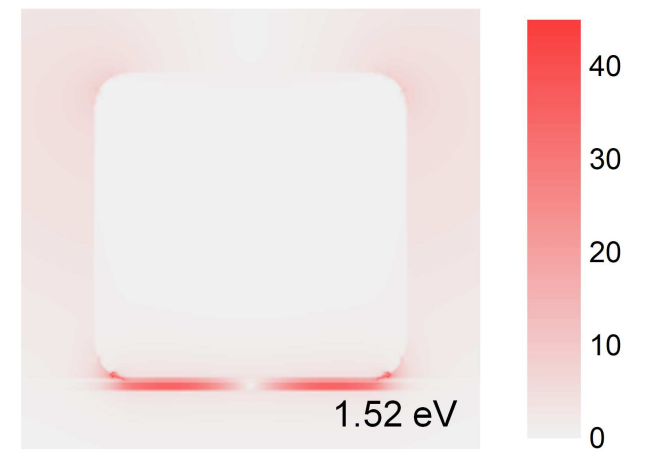

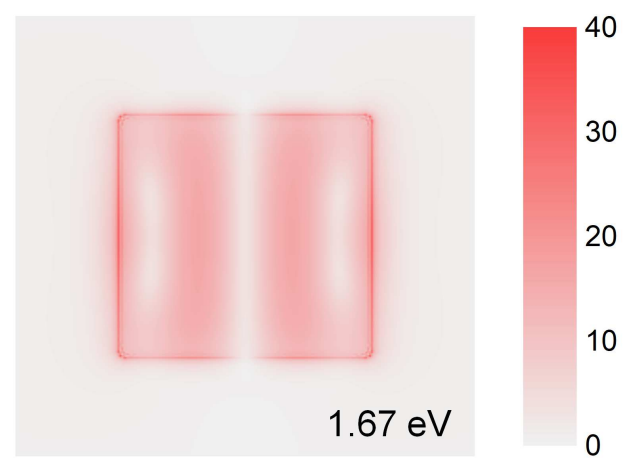

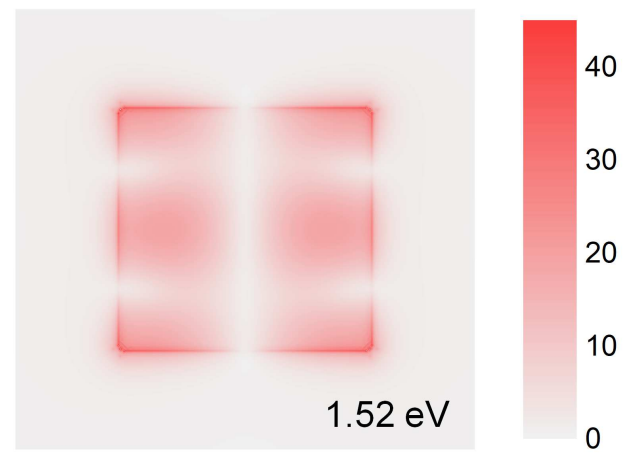

Figure 4

Mechanism of the enhanced upconversion of plasmonic cavity. (a) Schematic of the setup for collecting upconverted emissions in our experiments. (b), (c) Far-field angular radiation patterns for monolayer WSe2 in plasmonic cavity (b) and free space(c). (d), (e), (f) Charge and field distribution (|E/EO|) around plasmonic cavity for the mode at emitted photon energy $(1.67 \mathrm{eV})$. (g), (h), (i) Charge and field distribution (|E/EO|) around plasmonic cavity for the mode at excitation photon energy $(1.52 \mathrm{eV})$. 
a

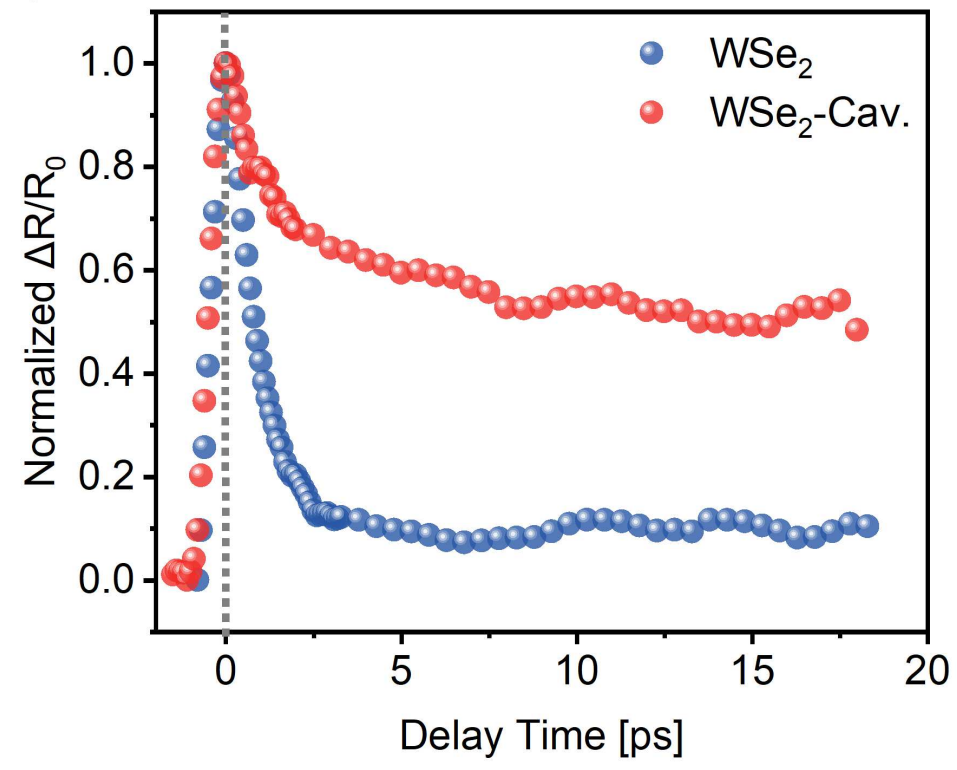

C

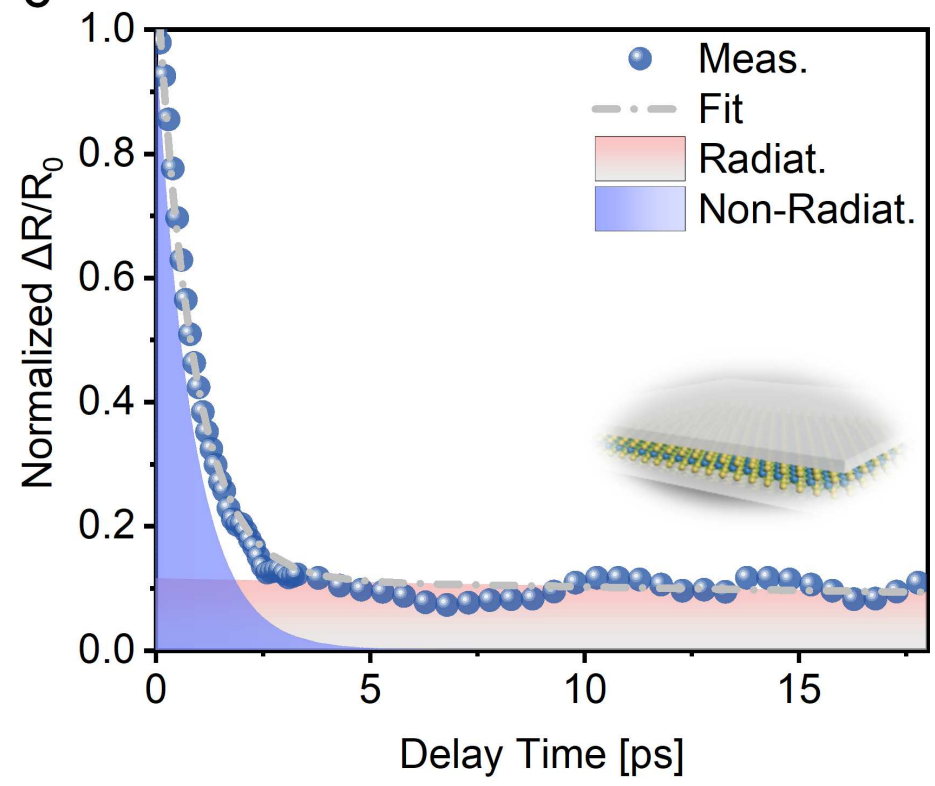

b

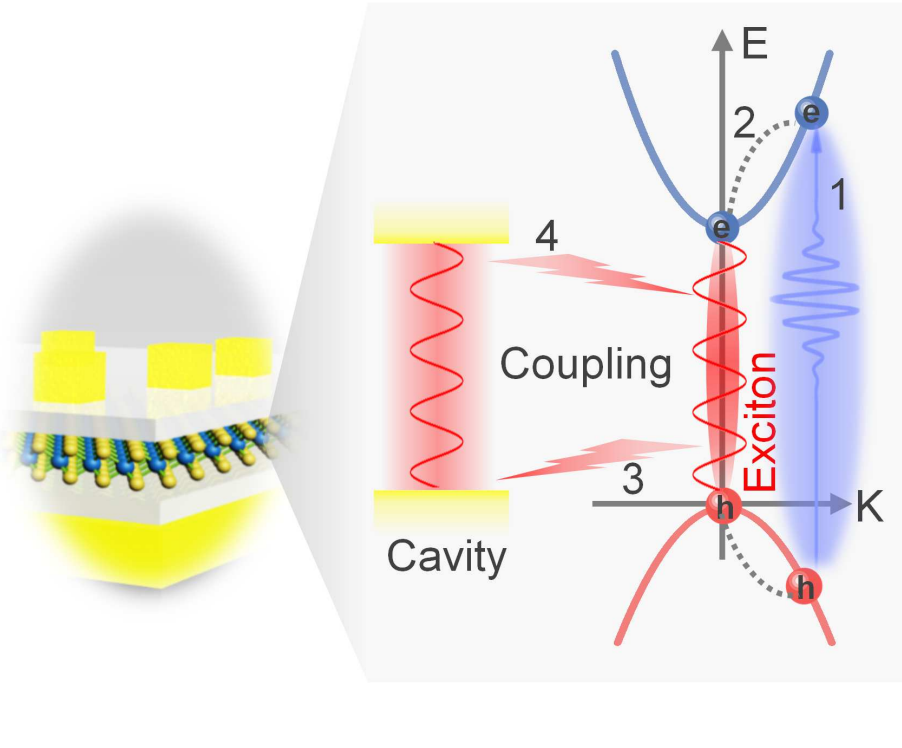

d

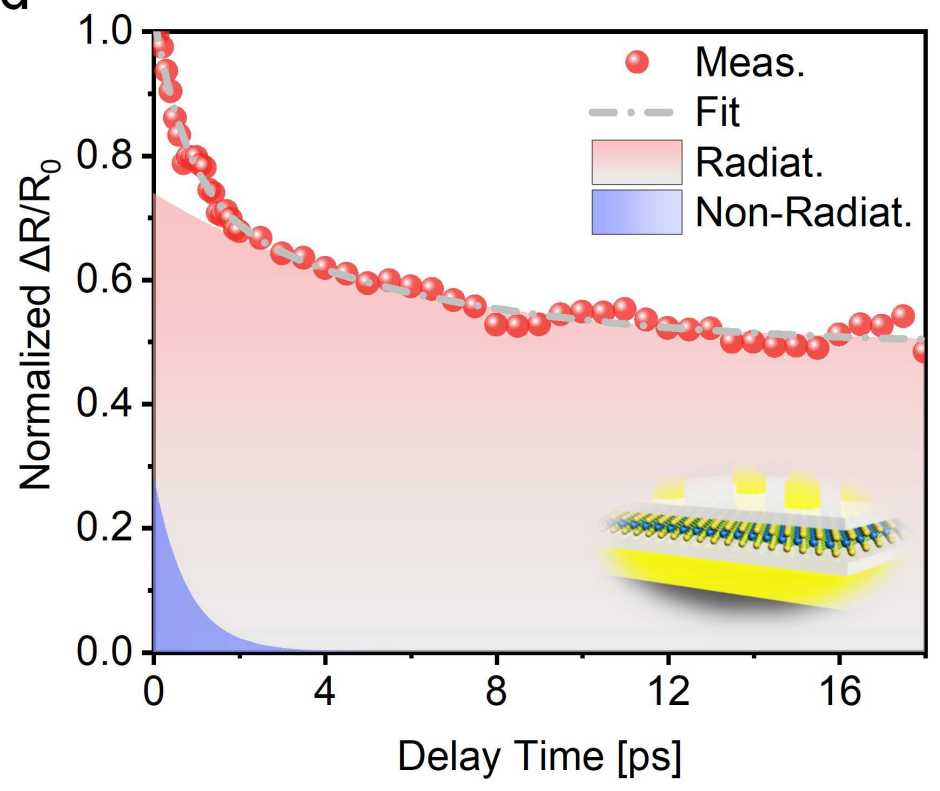

\section{Figure 5}

Purcell effect modulated exciton relaxation in plasmonic cavity. (a) Normalized differential reflection signal $\Delta R / R 0$ for the monolayer WSe2 in free space and plasmonic cavity. (b) Schematic diagram of purcell effect modulated exciton relaxation in plasmonic cavity: 1. Nonresonant excitation, 2. Intraband relaxation of energetic carriers, 3-4. Coupling between cavity and exciton emission. (c), (d) Fittings of the exciton relaxation in free space (c) and plasmonic cavity (d), respectively. 